\title{
The fine-scale structure of Long Beach, California, and its impact on ground motion acceleration
}

\author{
Jorge C. Castellanos ${ }^{1}$ and Robert W. Clayton ${ }^{1}$ \\ ${ }^{1}$ Seismological Laboratory, Department of Earth and Planetary Sciences, Caltech, Pasadena, California, \\ USA.
}

\section{Key Points:}

- We extract surface waves from the ambient noise recorded at three dense petroleum industry surveys that were deployed in Long Beach, CA.

- We make phase velocity measurements across multiple frequencies and invert for a 3-D shear wave velocity model of the region.

- We propagate synthetic wavefields through our velocity model to investigate the variability in the expected ground shaking intensity.

Corresponding author: Jorge C. Castellanos, jacastil@caltech.edu 


\begin{abstract}
The metropolitan Los Angeles region represents a zone of high-seismic risk due to its proximity to several fault systems, including the San Andreas fault. Adding to this problem is the fact that Los Angeles and its surrounding cities are built on top of soft sediments that tend to trap and amplify seismic waves generated by earthquakes. In this study, we use three dense petroleum industry surveys deployed in a 16x16-km area at Long Beach, California, to produce a high-resolution model of the top kilometer of the crust and investigate the influence of its structural variations on the amplification of seismic waves. Our velocity estimates reveal substantial lateral contrasts and correlate remarkably well with the geological background of the area, illuminating features such as the NewportInglewood fault, the Silverado aquifer, and the San Gabriel river. We then use computational modeling to show that the presence of these small-scale structures have a clear impact on the intensity of the expected shaking, and can cause ground-motion motion acceleration to change by several factors over a sub-kilometer horizontal scale. These results shed light onto the scale of variations that can be expected in this type of tectonic settings and highlight the importance of resolution in modern-day seismic hazard estimates.
\end{abstract}

\title{
1 Introduction
}

Los Angeles county and its surrounding areas are know for their susceptibility to earthquake shaking and for having several crossing faults that are capable of producing major damage to their infrastructure. The $1933 \mathrm{Mw}$ 6.4 Long Beach earthquake, for instance, caused widespread damage throughout the southern part of Los Angeles basin, resulting in more than 100 civilian fatalities (Wood, 1933) and over 40 million dollars in economic loss (Stover \& Coffman, 1993). The $1994 \mathrm{Mw} 6.7$ Northridge earthquake caused several gas leaks and collapsed numerous roadways and buildings across the region, making it one of the costliest disasters in US history (Bolin \& Stanford, 1998). More recently, the 2019 Ridgecrest earthquake sequence struck the Californian Mojave Desert, causing significant structural damage near its epicenters and noticeable shaking in the greater Los Angeles area.

Modern seismic hazard assessments are generally performed using deterministic approaches, in which a realistic representation of both the source process and the subsurface properties are used to obtain an estimate of the ground motion due to a large earth- 
quake (e.g., Costa et al., 1993; Wald \& Graves, 1998; Rodgers et al., 2018). As our knowledge of the crustal velocity distribution across southern California has evolved, these calculations have made it possible to identify zones in which the heaviest damage can be expected and have ultimately allowed the generation of building codes to make structures more resistant to earthquakes (Graves, 2008; Olsen et al., 2009; Graves \& Pitarka, 2016). However, with the ever increasing amount of seismic records, it has become clear that there are still significant differences between modeled and observed ground-motions across multiple scales that tend to produce large errors in seismic hazard estimates. Such discrepancies appear to stem from the fact that the shallow structure in Los Angeles basin is not well-characterized by the 3D models used in the simulations and that, as a result, its influence on the propagation of seismic waves is not entirely captured (Taborda et al., 2016; Wang \& Zhan, 2020). These shortcomings have important societal impacts as the underprediction of expected ground motion possess an immediate risk to the inhabitants of the area whereas the overprediction leads to costlier structural design and construction (Goulet et al., 2007).

Substantial work has been done in order to improve the accuracy of the velocity models of southern California. Examples of such efforts include the development of sophisticated imaging methods (e.g., Tape et al., 2009; Zhong \& Zhan, 2020; Muir \& Tsai, 2020), the incorporation of more seismic data (e.g., Magistrale et al., 2000; Kohler et al., 2003; Lee et al., 2014), and the deployment of temporary nodal arrays that allows us to explore the complex architecture of Los Angeles basin with refined detail (e.g., Liu et al., 2018). In particular, the introduction of oil-industry surveys into the field of crustal geophysics has dramatically increased the resolution of regional velocity models and, with it, improved the prediction of several seismic observables (Lin et al., 2013; Jia \& Clayton, 2021). Because of their unprecedented density, these seismic arrays are perhaps the most reliable way to determine the fine-scale structure of the crust and investigate the many complicated phenomena that can occur during seismic wave propagation. However, because of their financial cost, only a few regions around the world have hosted this type of experiments, resulting in a lack of knowledge on both the scale of structural variations and the spatial variability in shaking intensity that we can expect on this type of tectonic settings.

In this study, we use three of the highest resolution seismic arrays ever deployed in southern California to probe the elastic properties of the Long Beach crust and in- 
vestigate the seismic response of the ground beneath the surveys. Within this scope, we present a newly developed framework that allows us to perform reliable surface wave phase velocity measurements and construct a high-resolution 3-D model of the crust. We then report the peak ground motion acceleration that is expected at different sections of the experiment as obtained by propagating numerous wavefields through our velocity estimates. Finally, we discuss the potential of these type of arrays as well as their impact on future hazard assessment studies.

\section{Data and Methods}

\subsection{Ambient Noise Data}

Three dense arrays, with a total of $\sim 13,000$ seismic stations, were deployed as part of different petroleum industry surveys in Long Beach, CA, across the northwest-southeast trending Newport-Inglewood fault (NIF) system (Figure 1). Each of these networks (commonly known as the Long Beach, the Extended Long Beach and the Seal Beach arrays) consisted in a large group of 100-meter-equispaced high-frequency velocity sensors that were designed to illuminate the shallow oil deposits associated with the faulting of the area (Wright, 1991). Fortunately, during their time of operation, these instruments recorded continuously and, therefore, not only captured the active source component of the surveys, but also several passive sources including the ambient noise field. This last characteristic of the experiments allows us to move beyond the focus of traditional petroleum seismology and use this type of instrumentation to investigate local microseismicity (e.g., Li et al., 2018), the mechanics of active fault zones (e.g., Inbal et al., 2016), and the structure of the deeper crust (e.g., Lin et al., 2013; Nakata et al., 2015; Castellanos et al., 2020; Clayton, 2020).

In recent years, ambient-noise tomography has become a well-established imaging tool for investigating the elastic properties of the subsurface. This technique starts by cross-correlating the Earth's background vibrations recorded at a pair of receivers to approximate the Green's function between the two instruments (Shapiro \& Campillo, 2004; Shapiro et al., 2005; Bensen et al., 2007). Once a sufficiently long segment of ambient noise is cross-correlated, and the correlation function converges, classical tomographic techniques are applied to the emerged signals to extract critical information of the average velocity structure of the Earth along the path that waves have traveled (Yao et 
al., 2006; Moschetti et al., 2007; Lin et al., 2008; Ritzwoller et al., 2011). Because the resolution of ambient-noise tomography depends only on the geometry and distribution of seismic instruments, the analysis of noise-derived ballistic waves has enabled the construction of velocity models with unprecedented detail, and even allowed the mapping of the near-surface structure of seismically quiescent areas (e.g., Nicolson et al., 2012).

Here, we expand on the work of Lin et al. (2013) and use ambient noise data from all three temporary oil industry surveys to extract the Green's function between nodal instruments. Because these arrays operated at different times and, therefore, recorded asynchronously, each network was only cross-correlated with itself. The processing scheme used to process the noise recordings closely follows that of Bensen et al. (2007) and resulted in more than 30 million correlograms that show clear surface waves traveling between the different pairs of stations (Figure 2). Since the dense arrays were composed of vertical geophones only, the emerged signals likely correspond to Rayleigh waves. These arrivals are the base of our investigation and are the tool with which we image the structure beneath the arrays.

\subsection{Inversion for a 1-D Velocity Model}

As with every structural investigation, it is desirable have some a-priori knowledge of the average velocity structure of the target area before characterizing the small deviations from it. To this end, we use all of the available cross-correlations to generate a stacked gather for each of the surveys. We then convert these record sections to the Tau-p domain via a slant-stack scheme to obtain the frequency-phase-velocity representation of the signals (McMechan \& Yedlin, 1981). The collective analysis of multiple-offset traces allows to retrieve clear fundamental mode (FM) and first overtone (FO) dispersion curves for the $0.5-4 \mathrm{~Hz}$ frequency band, which we use to construct a shear-wave velocity profile that represents the mean structure of the top kilometer of the crust beneath the arrays. For the actual inversion, we average all of the extracted phase velocities to build a single FM and FO dispersion curve, and use Haney and Tsai (2017) perturbational algorithm to jointly invert the two modes for the best-fitting shear-wave velocity model. The average correlation gather, dispersion curves, modes sensitivities, and inverted velocity profile are shown in Figure 3a-e. 


\subsection{Surface Wave Relative Traveltime Measurements}

Although the slant-stack analysis of entire record sections allows us to retrieve robust velocity estimates, as evidenced by the agreement between our inversion results with the CVM-S4 reference model (Figure 3e), our ultimate goal is to analyze the small velocity variations across the seismic arrays. Such task, however, relies on our capability to extract reliable velocity measurements from individual cross-correlation functions, which can be problematic due to scattering, attenuation and the inherent high-noise level of the signals. For this reason, we implement an automatic neighborhood-based cross-correlation method for phase arrival picking that allows us to deal with complicated waveforms (Gouédard et al., 2012; Jin \& Gaherty, 2015). This technique begins by band-pass filtering all of the existing traces of a given source to a particular narrow-band frequency and measuring their differential delay times, $\Delta t$, with respect to the waveforms recorded at neighboring stations (Figure 4). To perform this calculation, we window the correlograms around the expected arrival time of the ballistic wave using a cosine-tapered window that is flat for 5 times the center frequency of the band-pass filter. Moreover, to address the problem of cycle-skipping, we limit the radius of the interstation cross-correlation to one wavelength long, as determined by the reference dispersion curve that was extracted from the stacked correlation gather in Figure 3. Once all of the cross-correlation derived differential delay times are collected, we arrange them into the form:

$$
\left[\begin{array}{ccccc}
1 & -1 & 0 & \cdots & 0 \\
1 & 0 & -1 & \cdots & 0 \\
1 & 0 & 0 & \cdots & 0 \\
\vdots & \vdots & \vdots & \ddots & \vdots \\
1 & 0 & 0 & \cdots & -1 \\
0 & 1 & -1 & \cdots & 0 \\
\vdots & \vdots & \vdots & \ddots & \vdots \\
1 & 1 & 1 & \cdots & 1
\end{array}\right]\left[\begin{array}{c}
t_{R_{1}} \\
t_{R_{2}} \\
t_{R_{3}} \\
t_{R_{4}} \\
t_{R_{5}} \\
t_{R_{6}} \\
t_{R_{7}} \\
\vdots \\
t_{R_{N}}
\end{array}\right]=\left[\begin{array}{c}
\Delta t_{R_{1} R_{2}} \\
\Delta t_{R_{1} R_{3}} \\
\Delta t_{R_{1} R_{4}} \\
\vdots \\
\Delta t_{R_{1} R_{N}} \\
\Delta t_{R_{2} R_{3}} \\
\vdots \\
0
\end{array}\right],
$$

so that the relative arrival time, $t$, of the wavefront recorded by every $R_{N}$ receivers can be inverted using a simple least squares method (VanDecar \& Crosson, 1990). To ensure that the traveltime field that is obtained from this operation is locally smooth, we introduce the regularization term: 


$$
\begin{gathered}
S\left(\mathbf{r}, \mathbf{r}^{\prime}\right)=\exp \left(-\frac{\left|\mathbf{r}-\mathbf{r}^{\prime}\right|}{2 \sigma^{2}}\right), \\
\int_{S} S\left(\mathbf{r}, \mathbf{r}^{\prime}\right) d \mathbf{r}^{\prime}=1
\end{gathered}
$$

where $\mathbf{r}$ is the position vector of the stations and $\sigma$ is the spatial smoothing width or correlation length (Barmin et al., 2001). Here, we set the value of this term to a half-wavelength of the filter's center frequency, and determine the overall strength of the smoothing kernel, $S$, from an L-curve analysis (Figure 5a). For the actual inversion, we chose to consider only high-quality waveforms and remove all relative delay times with a correlation coefficient smaller than 0.90. Figure 5b-c shows the inverted relative traveltime measurements for the 1-s period fundamental model Rayleigh wave that is derived from a single virtual source located at the northwestern end of the Long Beach array. Note that, to honor the far-field approximation, we only solve for the relative traveltimes of stations that are more than three wavelengths away from the virtual source (Yao et al., 2006; Lin et al., 2008).

To produce 3-D velocity model of the subsurface, it is necessary to characterize the propagation properties of surface waves that are sampling different depths of the crust. This task, in turn, requires us to extend our analysis to a wide range of frequencies, which can be challenging particularly when moving to a higher spectrum. For this reason, we introduce an intermediate step into our processing scheme, where we use the differential delay times of the long period waveforms to track the correct phase delays to higher frequencies (Figure 5d). This implementation allows us to use simpler signals to guide the relative traveltime surfaces to higher frequencies and hence avoid cycle-skipping.

In this study, we apply this algorithm to every virtual source available in all three of temporary surveys to generate relative traveltime surfaces of the fundamental mode Rayleigh waves for the $0.5-4 \mathrm{~Hz}$ frequency band. For these calculations, we used the 1s measurements to track the correct phase delay to higher frequencies, and performed an individual inversion for all the longer periods. We also disregarded the first-overtone surface waves since these wave packets were not always visible in individual waveforms and, for certain offsets and frequencies, the two modes interfered with each other, with the fundamental mode dominating the first overtone. Lastly, we remove all data involving the 300 marine nodes of the Seal Beach array as these recordings were particularly 
noisy and no clear surface waves from these virtual sources were observed. This operation resulted in almost 13,000 relative traveltime surfaces for each frequency that was analyzed, which we then used to construct phase velocity maps.

\subsection{Surface Wave Phase Velocity Measurements}

We use the collection of the relative traveltimes, to invert for the frequency-dependent phase velocities. To do so, we implement a time-based beamforming scheme, where, for a given virtual source, we take groups of relative arrival times that are less than a halfwavelength away from a given reference receiver and use a plane-wave approximation to solve for the horizontal slowness vector, $\mathbf{u}$, that can best explain the observations (Figure $6 \mathrm{a}-\mathrm{b})$. Here, we define said vector as:

$$
\mathbf{u}=u\left[\begin{array}{c}
\cos (\theta) \\
\sin (\theta)
\end{array}\right]=\left[\begin{array}{l}
u_{x} \\
u_{y}
\end{array}\right],
$$

where $u$ represents the horizontal slowness and $\theta$ the direction of propagation of the wave. From this expression, we can then write the relative arrival time of a group of receivers as:

$$
t(\mathbf{x}, \mathbf{u})=\mathbf{u} \cdot\left(\mathbf{x}-\mathbf{x}_{c}\right)
$$

where the vector $\mathbf{x}$ represents the spatial coordinates of every station in the subarray and $\mathrm{x}_{c}$ the center coordinates of the subarray. This formulation allows us to directly solve for the local phase velocity and the wave's propagation direction at the different sections of the array with very few assumptions. However, the primary reason why we measure phase velocities with this approach is because this operation is efficient enough to allow the use of Gaussian statistics to quantify the robustness of the inverted slownesses and azimuths. To do so, we apply a bootstrapping method to every subarray relative traveltimes using a total of 300 resamples and, for each family of measurements, obtain a new local phase velocity and azimuth (Figure 6c-d). We then translate the spread of these quantities into an absolute error value by taking the product of the eigenvalues of their covariance matrix. To show the performance of our scheme, Figure 6e-g shows the local phase velocity, backazimuth, and associated error for the 1-s period Rayleigh wave that is derived from a single virtual source located at the southeastern end of the Long 
Beach array. It is worth noticing how the stations with the largest error are coincident with the sections of the survey that have a relatively anomalous phase velocity and backazimuth estimation.

Once all of the virtual sources have been used to derive the frequency-dependent phase velocity measurements, we weight-stack them to produce an isotropic phase velocity map. For this process, we use the bootstrap-derived errors as a mean to weight the phase velocity values and opt to only retain sites in which more than 100 velocity measurements were made. This step allows us to dramatically reduce the effects of irregular measurements and obtain a robust representation of the phase velocity at each site. Figure 7 schematically shows the construction process of the 1-s period isotropic phase velocity map of the Long Beach array, together with a group of higher-frequency velocity maps that were generated using the longer period relative phase delay times as reference.

Up to this point, all of the processing steps and examples that have been presented here have been derived using data from the Long Beach survey. Nonetheless, the adaptability of our framework makes it straightforward to extend our analysis to the neighboring arrays. Figure 8 shows the phase velocity distribution for the 1-s period Rayleigh wave that is obtained by combining all three of the temporary surveys. From this image, we can note the continuity of the phase velocity estimates, as evidenced by the northwestsoutheast trending fast anomaly that is characteristic of the NIF (Lin et al., 2013). This feature of our velocity map suggests consistency in our processing scheme. Moreover, we compare our phase velocity measurements across the Seal Beach array with a reflective time slice that was provided by the oil company that processed the active source data (Gish \& Boljen, 2021). From this comparison, we find that there is a remarkable agreement between our velocity estimates and the main reflection horizons, particularly those associated to the Garden Grove Fault and the prograding clinoforms located near the center of the survey. To show the comparison of these two quantities in a more dynamic manner, Video S4 shows a progressive overlay of the reflective image on the 1-s phase velocity map. 


\subsection{Inversion for Shear Wave Velocity}

After all of the velocity maps are constructed, we extract a regionalized dispersion curve at each point across the survey and use the Haney and Tsai (2017) perturbational algorithm to map the phase velocity as a function of frequency to shear wave velocity as a function of depth. For each inversion, we use a homogeneous velocity profile that is discretized into 25-m layers to iteratively update each component of the model until a satisfactory fit to the dispersion curve is achieved (Figure 9). Throughout this process, we assume a constant $V_{p} / V_{s}$ of 1.8 for the entire structure and determine the density from the compressional wave velocity using the empirical relationship of Gardner et al. (1974). Each velocity profile is then integrated into a whole 3 -D velocity model that represents the structure of the top kilometer of the Long Beach crust.

\section{Results and Discussion}

Figure 10a shows three depth-slices of the shear wave velocity model that is obtained through the inversion of the regionalized dispersion curves. It is clear from these images, that there are significant variations in the velocity structure beneath the surveys. At shallow depths $(<100 \mathrm{~m})$, the range of velocity variations across the survey is close to $10 \%$, with the slower velocities being concentrated in the southern part of the Long Beach array and around the San Gabriel river. In these images the Silverado water bearing unit and a section of the Compton-Los Alamitos Fault system can be seen. Both of these structures are of great importance of the area as the former is the main supplier of the groundwater that is extracted in Long Beach (Poland, 1956), and the latter is part of a blind thrust fault that is known to be capable of generating large magnitude earthquakes (Mw 7.0-7.4; Leon et al., 2009).

At deeper depths $(\sim 600 \mathrm{~m})$, the most prominent feature in our velocity maps is the NIF. In agreement with Lin et al. (2013), we argue that the fast velocity signature of this structure is related to the presence of deeper (and hence faster) rocks that were exhumed by the transpressional stresses that are acting on this region (Wright, 1991; Wesnousky, 2005). This deformation process would also explain why the migrated reflection images generated by oil company that carried out the seismic survey delineate the NIF as a flower-shaped damaged-zone rather than a single planar boundary that extends continuously to the deep crust (Figure 10b). The identification of this type of structures is 
relevant not only because they hold the potential of forming profitable hydrocarbon traps (Harding, 1985), but also because they can provide important insights into the type and extent of faulting that we can expect in a particular area (Inbal et al., 2016; Yang et al., 2021).

As a final product of the surface-wave analysis, we present an azimuthal anisotropy model for the structure beneath the surveys. This quantity describes how the velocity of a particular wave varies with its direction of propagation which, for the very shallow earth, is often caused by the preferred alignment of cracks by the regional tectonic forces (Crampin, 1994). The analysis of anisotropy can thus illuminate the direction of the stress field and potentially detect faults that might be present in the area (e.g., Ozacar \& Zandt, 2009; Licciardi et al., 2018). Figure 11 shows how the phase velocities for the 1-s Rayleigh wave vary as a function of azimuth at two different sites of the Long Beach array. These variations display a strong directional dependence that can be well-described by the $2 \theta$ components of Smith and Dahlen (1973) model for surface wave phase velocity, $C$, in a weakly anisotropic media:

$$
C(T, \theta)=C_{0}(T)+C_{1}(T) \cos (2 \theta)+C_{2}(T) \sin (2 \theta)+C_{3}(T) \cos (4 \theta)+C_{4}(T) \sin (4 \theta),
$$

where $T$ represents the period, $\theta$ the backazimuth, $C_{0}$ the isotropic velocity, and $C_{1-4}$ the azimuthal coefficients (Backus, 1970). This parametrization allows us to translate the wavefield's azimuthal dependence into a fast direction term and an amplitude term, which we can then visualize on a horizontal plane to determine whether any spatial patterns exists, and what association they have with the regional geologic structures. Figure 16 shows how the anisotropy for the 1-s Rayleigh waves varies across surveys. These measurements are mostly sensitive to the 100 meters depth and exhibit a dominant northsouth orientation that is overall consistent with the maximum horizontal compressional direction of southern California (Yang \& Hauksson, 2013). However, with the resolution that is provided by the nodal arrays, we can now begin to illuminate sub-kilometer variations of the stress field, as is evident by the slight rotation of the anisotropy vectors just at the center of the NIF near Signal Hill (yellow star in Figure 12). This observation is consistent with the results of Lin et al. (2013), who perform a similar analysis with the Long Beach array data and suggested that there might be a small-scale stress change near the fault zone segmentation. 
With the modest improvements in our processing scheme, which permits the retrieval of velocity estimates with much less spatial averaging, we are able to detect and interpret even smaller changes in the anisotropy pattern that could potentially be related to unmapped faults or other geological boundaries (red dashed lines in Figure 12). To validate this claim, Figure 13 shows snapshots of a propagating wavefront that is obtained by cross-correlating the ambient noise data of the dense surveys with the broadband station LAF from the SCSN (see Figure 1 for its location). The path to this particular instrument is oriented parallel to most tectonic faults in the area (relative to the port of Long Beach) and is thus ideal to detect the presence of any geologic structures that have a similar strike. This figure shows that there exist large lateral variations on the propagation properties of the wavefield and that the wavefront appears to be "cut" just along the planes where our anisotropy measurements suggest the presence of a structural barrier (cyan dashed lines in Figure 13). This feature is largely evident at propagation time of 45 seconds, where wavefront is deflected just at the boundary where our anisotropy vectors display a dramatic change in orientation, at the site where the seismic reflection image marks the location of the Garden Grove Fault (see reflection image in Figure 8). These results suggest that the analysis of azimuthal anisotropy has the potential of detecting sharp structural boundaries.

With the robust velocity estimates that were derived from our ambient noise analysis, it is now possible to investigate the effects of the fine-scale structure on ground motion acceleration. For this endeavor, we propagate realistic 3-D wavefields through our high-resolution velocity model using Salvus, a high-performance spectral-element solver (Afanasiev et al., 2019), and calculate the amount of shaking that is expected on different parts of the survey. The peak ground acceleration (PGA) that is obtained in this step is then compared to the one that results from propagating an equivalent wavefield through the 1-D model that was obtained from the inversion of the average dispersion curves in (Figure 3b). This approach, in principle, allows us to characterize the influence of the local geological structure on the amplitude of the seismic waves and separate the influence of factors such as the source radiation pattern and geometric spreading from the response.

To provide a concrete example of the above, we place a vertical point source at the surface on the north-western end of the Long Beach survey, just a-top of the NIF, and record the entire time evolution of the wavefield (Figure 14). Here, because of the choice 
of source geometry and configuration, most of the energy that is inserted into the system will propagate in the form of surface waves (Harkrider, 1964). This exercise allows us to see the largely asymmetric shape of the wavefront and reveals the existence of significant lateral variations on the magnitude of acceleration as the energy propagates away from the source. Such variations are particularly obvious in Figure 15, where we present the PGA ratio that is obtained by comparing our estimates with those obtained from the 1-D modeling. From this analysis, we can quantitatively observe how the small-scale variations in the shallow velocities can produce rapid changes in the vertical PGA and, most interestingly, how the structure of the NIF can generate elongated zones of intense amplification that result from the focusing and self-intersection of the propagating wavefront (i.e. from multipathing). These findings are in remarkable agreement with those of Clayton et al. (2011), who used data from a nearby earthquake to document that the velocity contrasts underneath the Long Beach array can cause ground accelerations to vary by a factor of five over a horizontal scale length of one kilometer.

The results in Figure 15 show that near-surface velocity variations can significantly alter the propagation properties of earthquake wavefields. To quantify this phenomena, we determine the amount of shaking that is expected from a vertically incident shear wave by placing an earthquake source beneath each station of the survey and propagating them to the surface (e.g., Wills et al., 2000; Bowden \& Tsai, 2017; Jia \& Clayton, 2021). For a source, we use a horizontal force injected by a $2 \mathrm{~Hz}$ Ricker wavelet located at 2-km depth (Figure 17). We then compare these estimates with the ones that are obtained from an equivalent 1-D run to obtain a measure of the relative amplification that occurs at each site. We record the three-component acceleration and calculate the PGA from the magnitude of acceleration to account for any non-vertical propagation that might occur due to the heterogeneous 3-D structure. The amplification measurements that are obtained from this exercise are presented in Figure 17.

The amplitude of variation in the amplification factors that we obtain through this analysis is smaller than the one we observe from a distant source. However, it is important to note that these measurements are meant to look at the amount of local amplification at each individual site without taking into consideration the integrated effects from other lateral heterogeneities. Within this context, we observe that the largest amount of amplification can be expected at the northwest and southeast sections of the survey, and that the area with the least amount of amplification is concentrated around the NIF. 
This result is theoretically-consistent with what is expected since, as mentioned above, the NIF zone appears to be composed of faster earth materials and, therefore, has stiffer site conditions. The standard quantity that is used to determine near-surface amplification is Vs30, the shear wave velocity in the top $30 \mathrm{~m}$ of the crust (Thompson et al., 2014). In Figure 18, we show the velocity variations averaged from the top two layers in our model (top $25 \mathrm{~m}$ ) and is about a factor of two higher, which is likely due to averaging from deeper layers as indicated by the sensitivity kernel show in the figure. However, the biggest difference is in the contribution from the topographic slope component of the USGS estimate. This is dominated by the small compact edifice of Signal Hill, which rises 100-m above the surrounding area, and is pop-up structure caused by convergence across two strands of the Newport-Inglewood Fault. This comparison suggests that Vs30 may not be a useful measure of amplification in the Long Beach area.

\section{Conclusions}

In this study, we used ambient noise data recorded at three dense petroleum industry surveys that were deployed in Long Beach, California, to map the small-scale heterogeneities of the crust and investigate their impact on the amplification of seismic waves. For this purpose, we developed a fully-automatic neighborhood cross-correlation method for phase arrival picking that allowed us to perform robust phase velocity measurements across a wide frequency range. We then used these dispersion measurements, together with a time-based beamforming approach, to construct high-resolution phase velocity maps and invert for a 3-D shear wave velocity model of the top kilometer of the Long Beach crust. Our velocity estimates compare well with the structure image from the activesource reflection survey that was done with the array, and also show a strong correlation with the main geological features of the area. We also presented evidence that speak of the possibility of using seismic anisotropy observations to detect small-scale changes on the regional stress regime and illuminate faults whose traces are not necessarily visible at the surface. Lastly, we propagated synthetic wavefields through our velocity model to quantify the scale of variability and intensity of amplification that can be expected across the different parts of the experiments. For the case of surface waves, our results revealed that some of the fine-scale structures that are present in this area are capable of causing complicated wave phenomena that can result in rapid lateral changes on the intensity of shaking of over a factor of ten over a horizontal distance of less than one kilo- 
meter. For the case of vertically propagating shear-waves, our results suggest that the mildest shaking can be expected in the vicinity of the Newport-Inglewood fault, as the majority of the fault zone is composed by denser earth materials that were exhumed by the transpressional stresses that are acting on the region. In general, our results not only provide insights into the geo-mechanical properties of sedimentary basins but also highlight the enormous potential of dense nodal arrays to improve the reliability of modern hazard assessments.

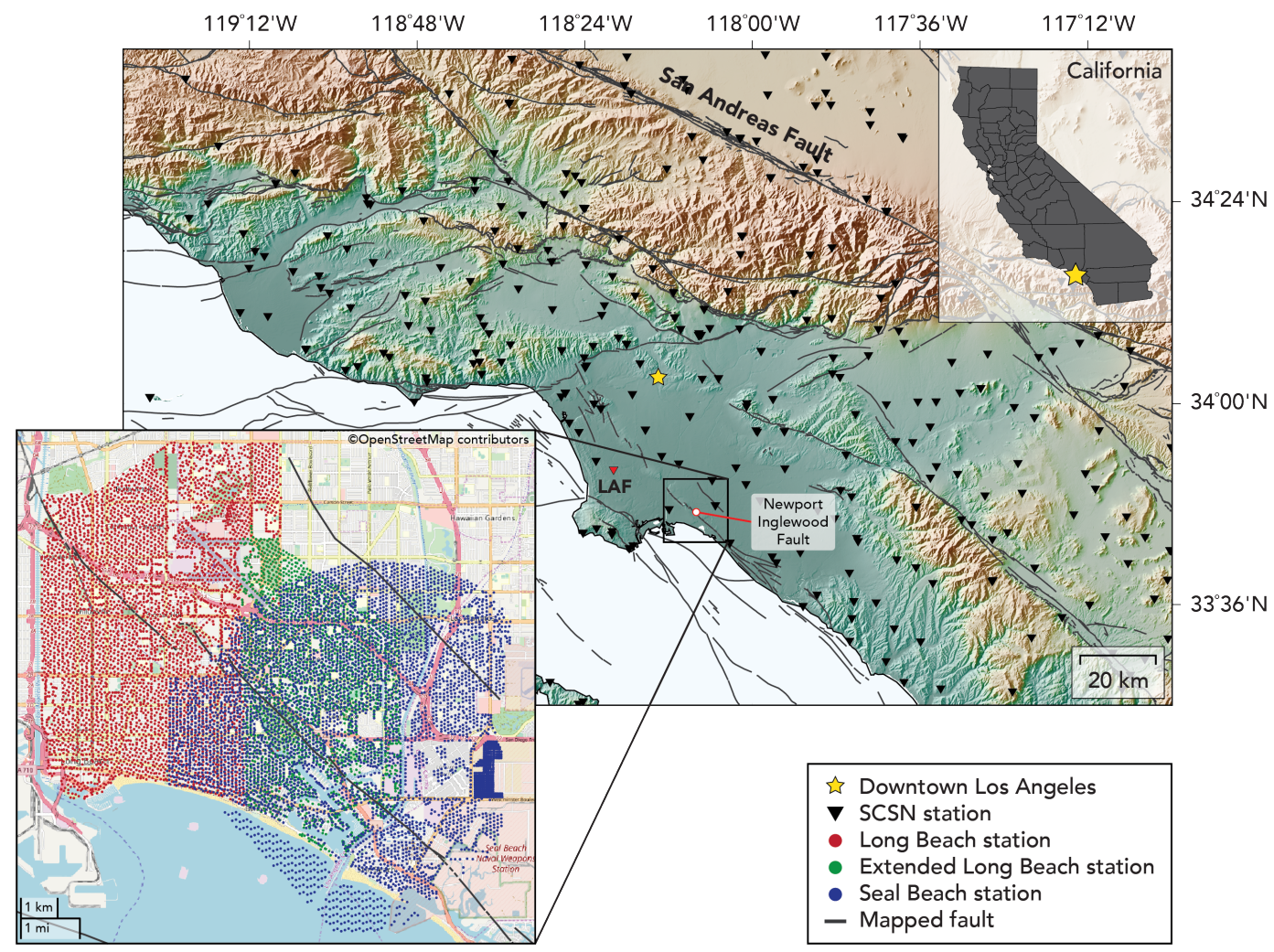

Figure 1. Regional map of southern California and the Continental Borderland. The black rectangle marks the area where the three petroleum industry surveys were deployed and the inset map shows the distribution of the instruments (with the Long Beach array in red, the Extended Long Beach in green, and the Seal Beach array in blue). The stations of the Southern California Seismic Network (SCSN) are shown as inverted triangles and the regional mapped faults are delineated with thick gray lines. Seismic station LAF is shown as an inverted red triangle. 

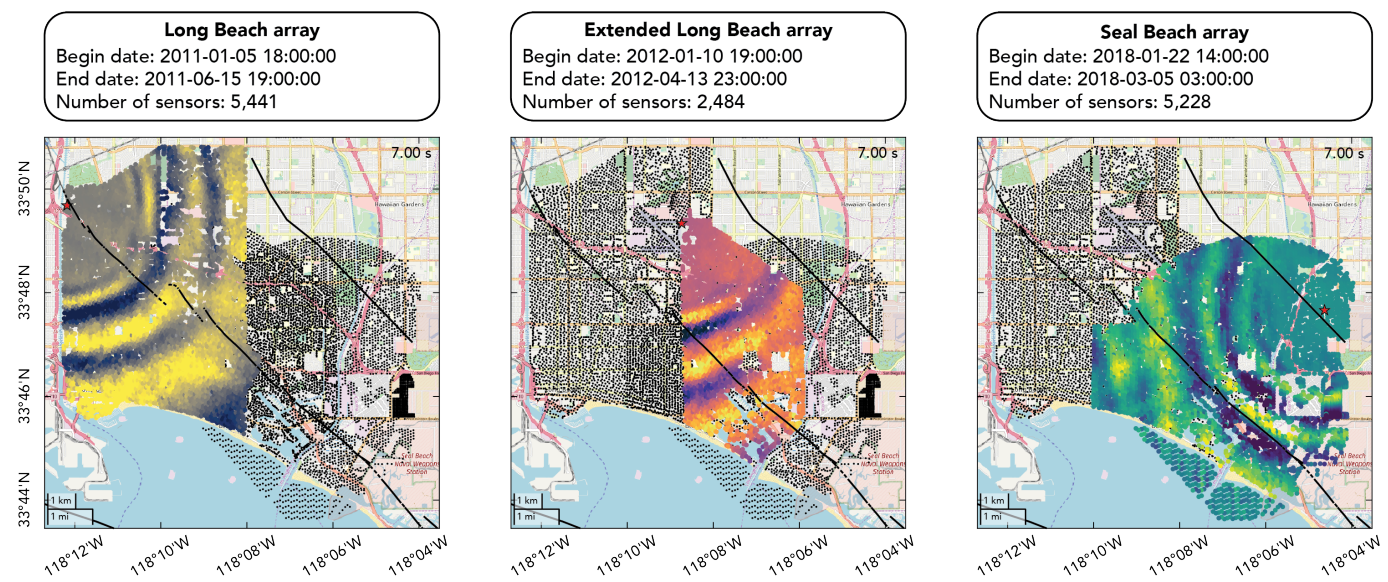

Figure 2. Wavefield snapshots of the ambient noise correlation functions from a single virtual source of the Long Beach array (left), Extended Long Beach array (middle), and the Seal Beach array (right). Clear ballistic waves propagating away from all three of the sources can be observed. The number of instruments and time of operation of each survey is presented on top of each panel. For the complete time evolution of the wavefields, the reader is referred to Videos S1-3. 

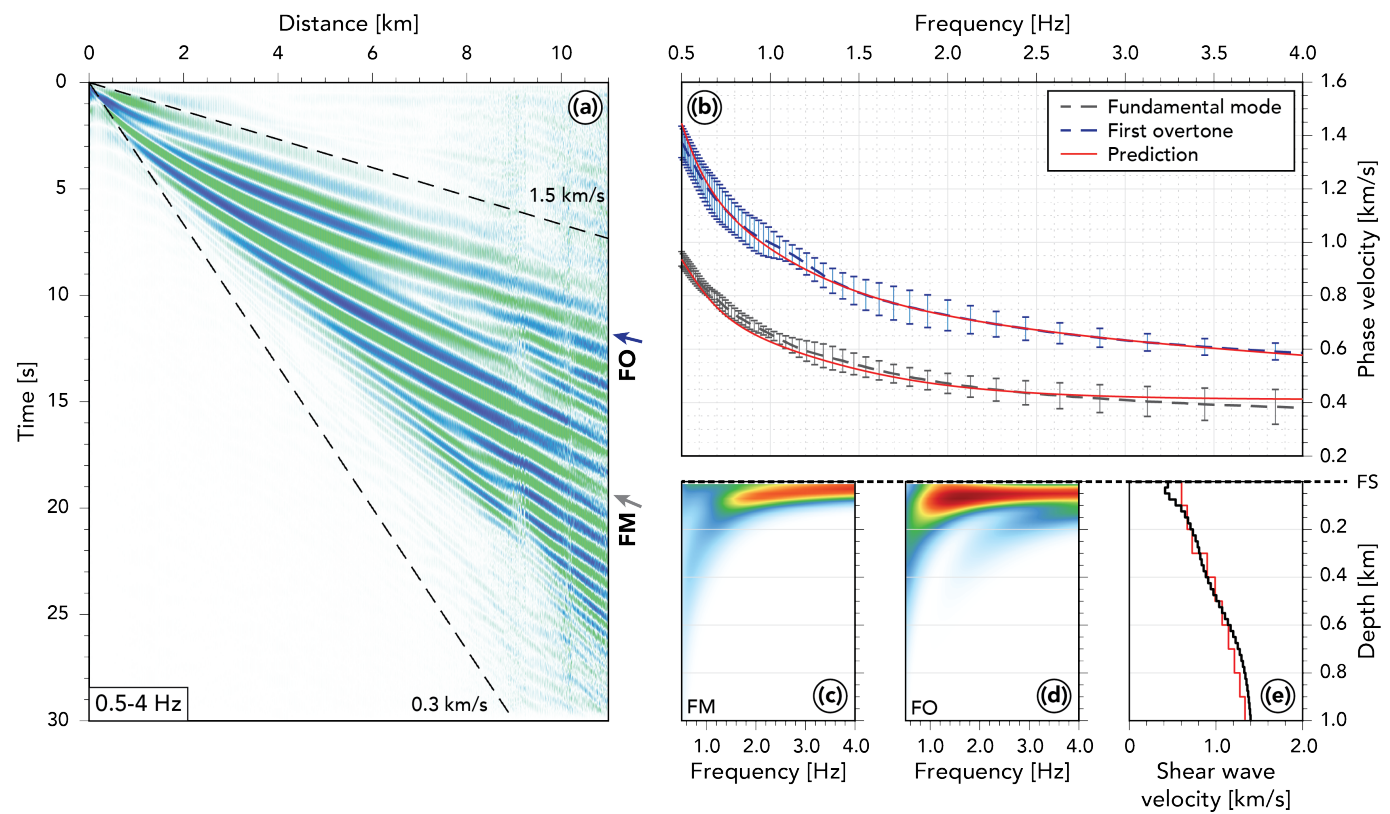

Figure 3. Stacked gather of the ambient noise cross-correlations and inversion for an average 1-D shear-wave velocity model. (a) Shows the first 30 seconds of the stacked cross-correlations from all three surveys. The bin-size of the spatial stacking is of 100 meters. The waveforms are band-passed filtered between 0.5 and $4 \mathrm{~Hz}$. Clear fundamental (FM) and first overtone (FO) surface waves traveling at velocities between 0.3 and $1.5 \mathrm{~km} / \mathrm{s}$ can be observed for the entire distance range. (b) Shows the average FM and FO dispersion curves that are obtained through the slant stack analysis of each survey's dataset. The continuous red lines represent the predicted dispersion curve that results from our inversion process. (c-d) Shows the sensitivity kernels for the FM and FO, respectively. (e) Shows a comparison between shear wave velocity profile that is obtained from jointly inverting dispersion curves in (b) (black line) and a profile that is extracted from the CVM-S4 model (Shaw et al., 2015) at the geographic center of the nodal stations (red line). The black dashed line on top of panels (c-e) represents the free surface (FS). 


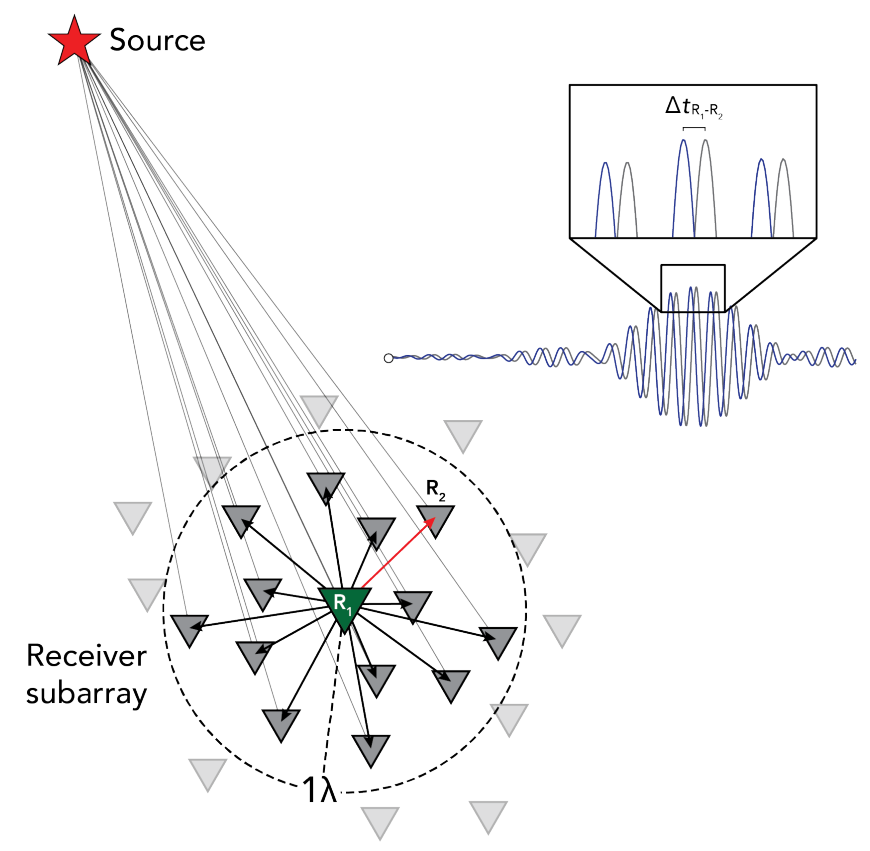

Figure 4. Schematic representation of the neighborhood-based cross-correlation method for phase arrival picking. Receivers that are less than one wavelength apart $(<1 \lambda)$ from a reference station $\left(R_{1}\right)$ are grouped together to form a subarray. The differential delay times, $\Delta t$, between the reference waveform and all the other waveforms in the subarray are measured through waveform cross-correlation. 

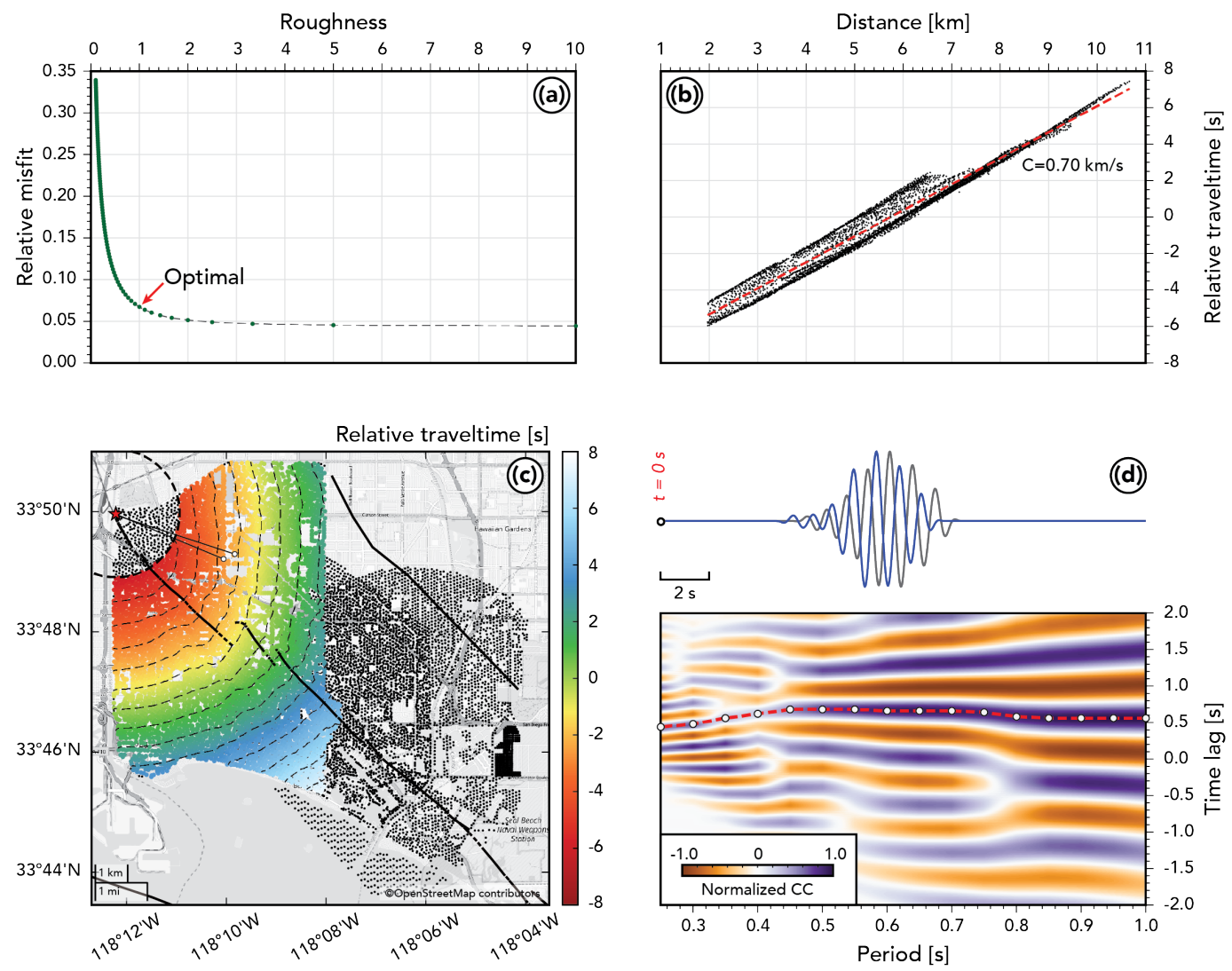

Figure 5. Example of relative phase delay measurements for a virtual source of the Long

Beach array. (a) Shows the L-curve analysis that was performed to determine the strength of the regularization term, $S$, in the inversion of the relative traveltimes. (b) Shows how the resulting relative traveltimes of the 1-s period waveforms vary as a function of distance from the virtual source. The dashed red line on top of the measurements has a corresponding slope of $0.70 \mathrm{~km} / \mathrm{s}$.

(c) Shows the spatial distribution of the relative traveltimes that are in (b). The contours are drawn in time increments of 1 second. The red star denotes the location of the virtual source and the thick dashed circle around the source marks the three-wavelength minimum distance criterion that was imposed in our analysis. (d) Shows the process by which we extend the differential delay measurements to higher frequencies. The waveforms on the top are two ambient noise cross-correlations functions that are filtered at 1-s period and windowed around the expected time of arrival of the ballistic surface waves. The source-receiver raypath of these two waveforms is shown in (c). The bottom panel shows the correlation function of the two traces as a function of period. To determine the differential delay time of these two waveforms across the entire frequency range, we use the 1-s measurement to track down the phase delay to higher frequencies (red dashed line). 

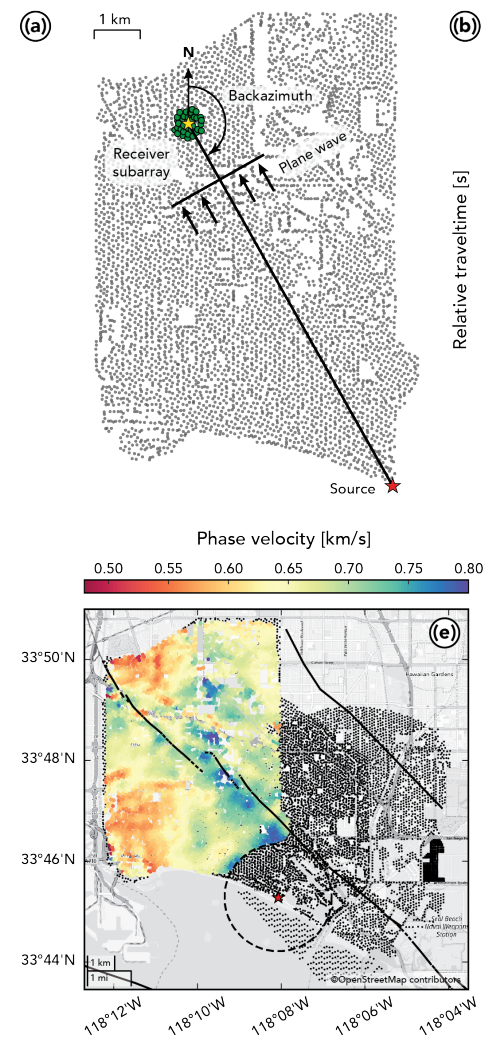
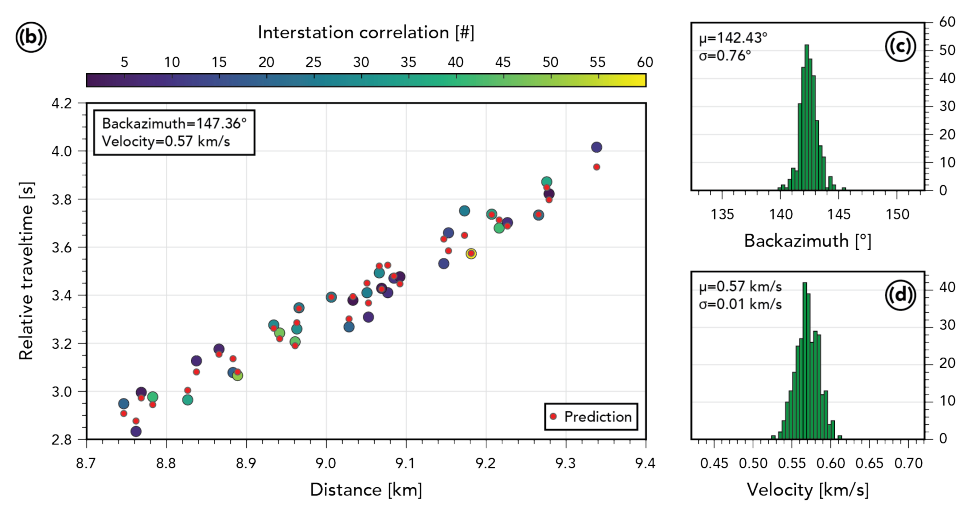

Backazimuth $\left[^{\circ}\right]$
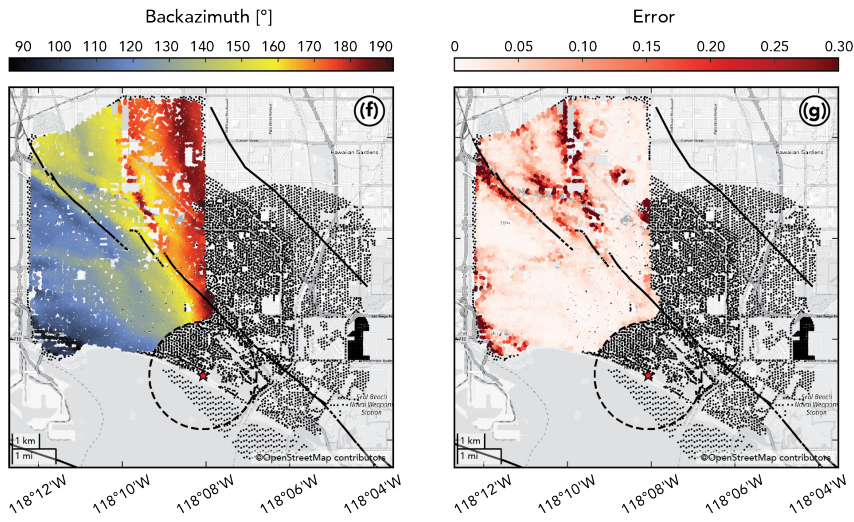

Figure 6. Example of phase velocity measurements for a virtual source of the Long Beach array at 1-s period. (a) Shows the source and receiver configuration that is used to make a single phase velocity measurement at a particular site. The yellow star marks the reference station that is used to form the receiver subarray and the green circles correspond to all the stations were grouped together using the half-a-wavelength maximum distance criterion. The aperture of the subarray is approximately one-wavelength long. (b) Shows the relative traveltime of the receiver subarray in (a) as a function of their distance from the virtual source. Each marker is color-coded by the amount of times that a differential measurement of that station was used to construct the relative traveltime surface. The red circles depict the predicted relative arrival time that result from our time-based beamforming. (c-d) Show the distribution of our bootstrapped backazimuth and velocity measurements for the data shown in (b). (e-g) Shows the spatial distribution of all the velocity, backazimuth and error measurements that were obtained for the same virtual source. 
(a)
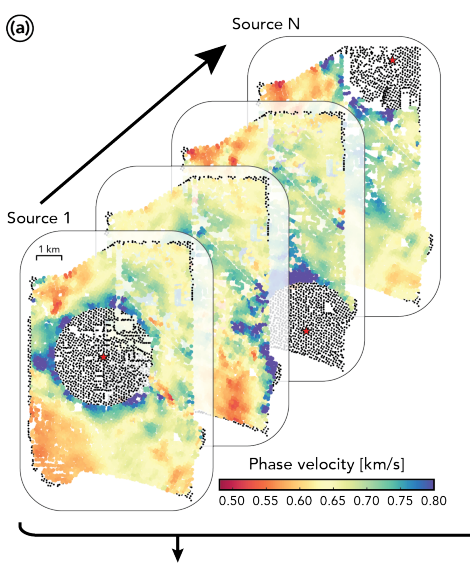

(b)
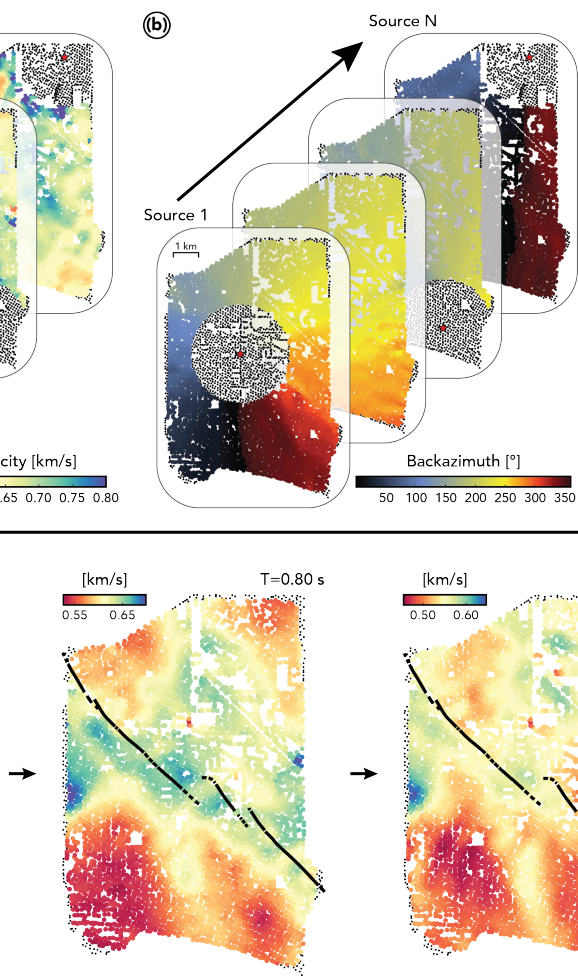
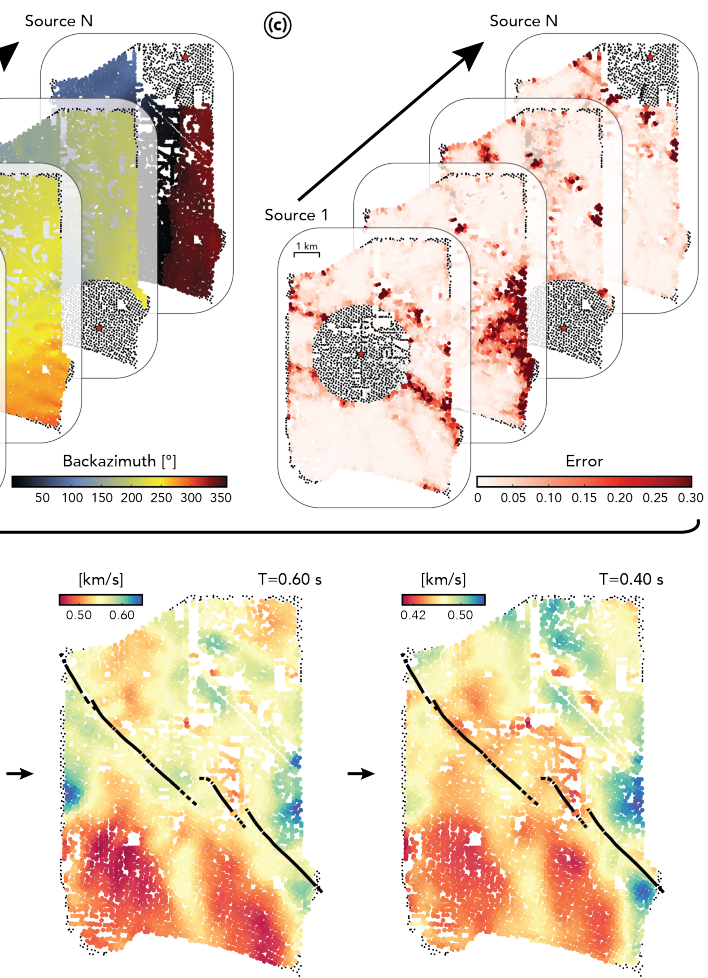

(di)

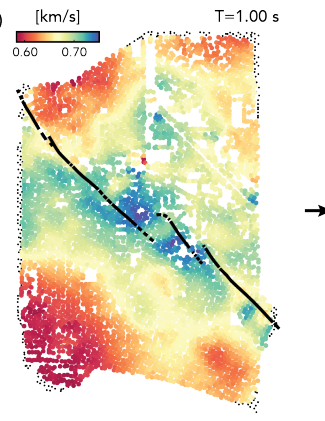

Figure 7. Schematic representation of the construction process of isotropic phase velocity maps. (a-c) Show the phase velocities, backazimuths and error measurements for different virtual sources of the Long Beach array at 1-s period. The errors in (c) are used to weight the averaging of the velocities in (a) to ultimately construct a frequency-dependent isotropic velocity map. The backazimuth measurements in (b) are reserved and used at a later step to characterize the anisotropy across the array. (d) Shows, from left to right, the isotropic phase velocity maps for the 1-, 0.8-, 0.6-, and 0.40-s Rayleigh waves. Each of these maps are generated in the same fashion, with the exception that the relative phase delay measurements of the higher frequencies were derived from the 1-s period waveforms. For periods larger than 1-s, such measurements were done independently. 


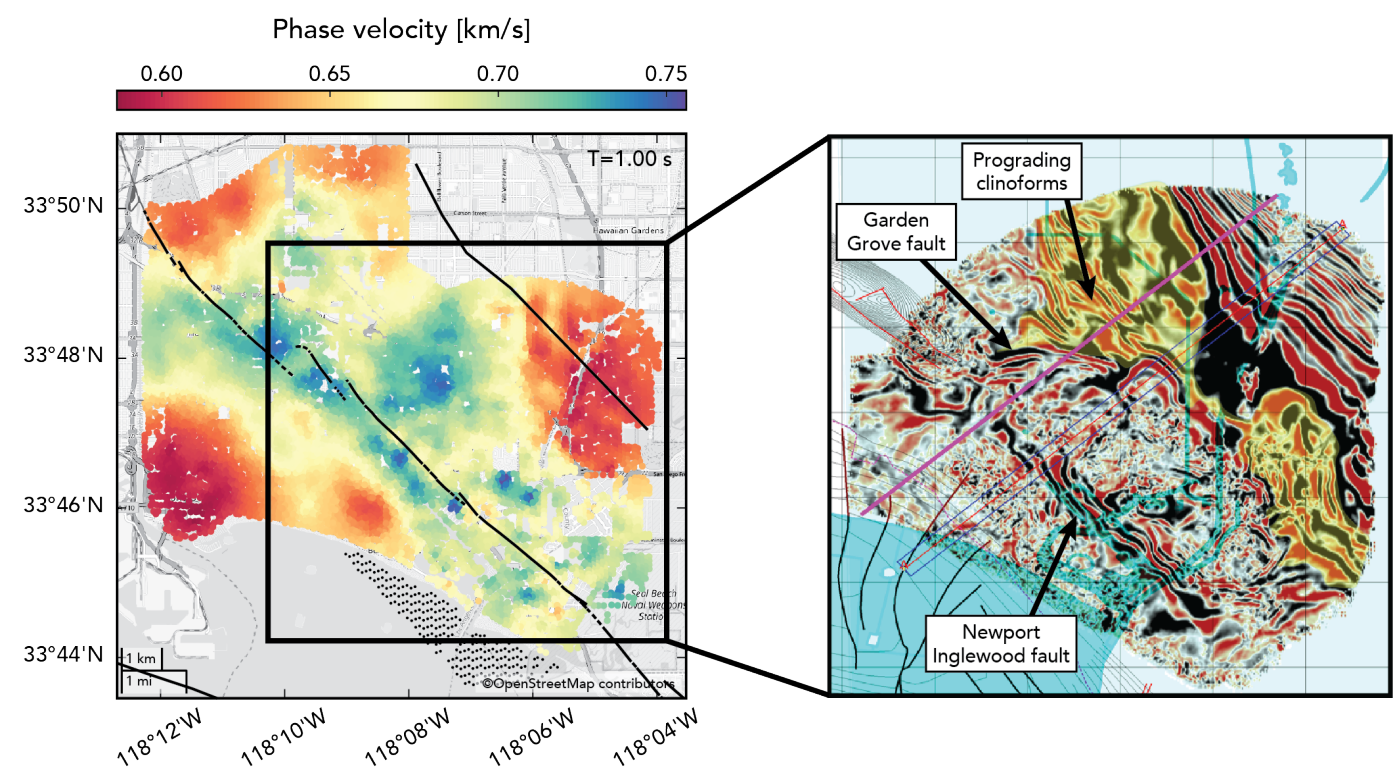

Figure 8. Comparison of our 1-s isotropic phase velocity map (left) with a reflective time slice of the active part of the survey at $0.40 \mathrm{~s}$ (right). Despite their difference in nature, the two different quantities in these images appear to illuminate similar tectonic features. The reflection image is courtesy of 3D Seismic Solutions. For a dynamic overlay of these two images, the reader is referred to Video S4. 

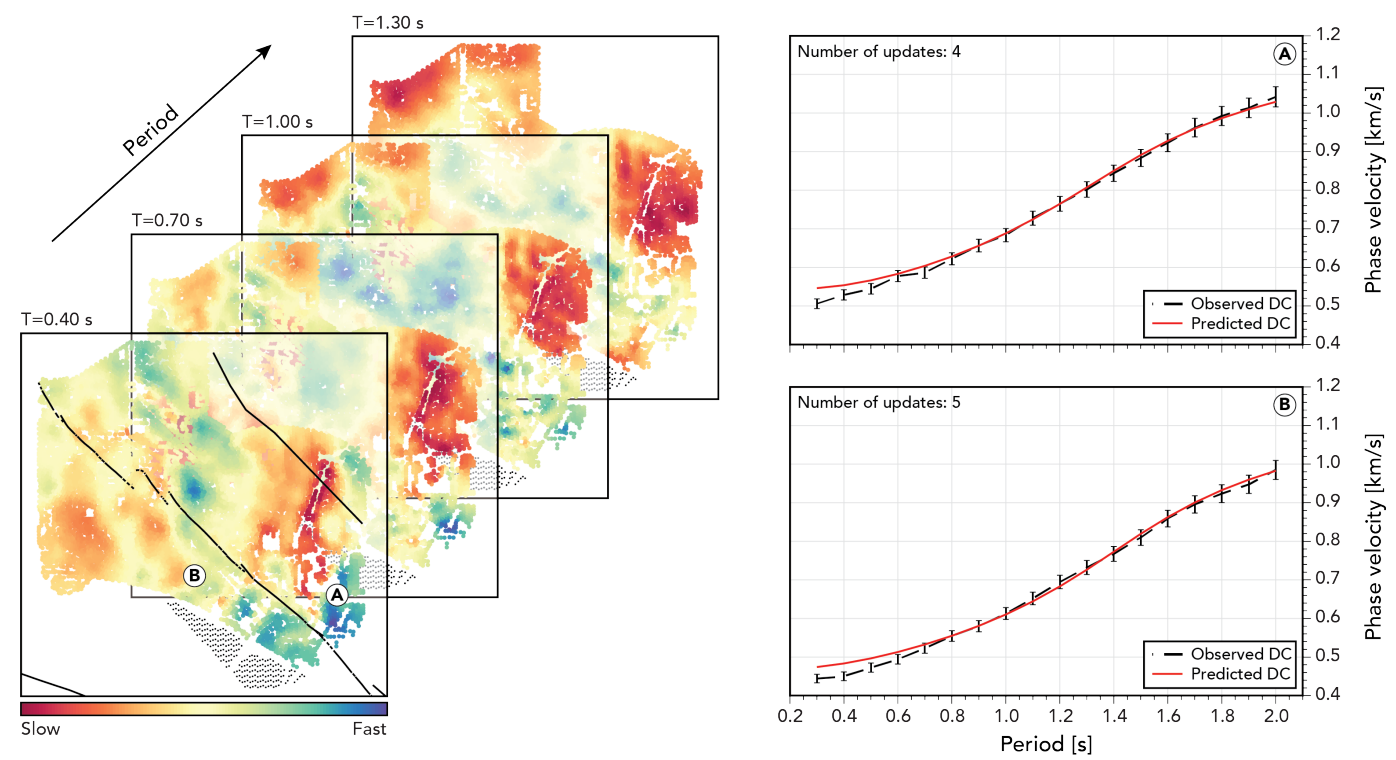

Figure 9. Inversion process of the regionalized dispersion curves. The maps on the left show how the phase velocities vary as a function of period. The black dashed black lines on the right panels correspond to the dispersion curves that are extracted at locations A and B of the maps. The red continuous lines correspond to the dispersion's best-fit. The number of updates that were required for the inversion to converge in these two examples are shown on the top-right of each panel. 

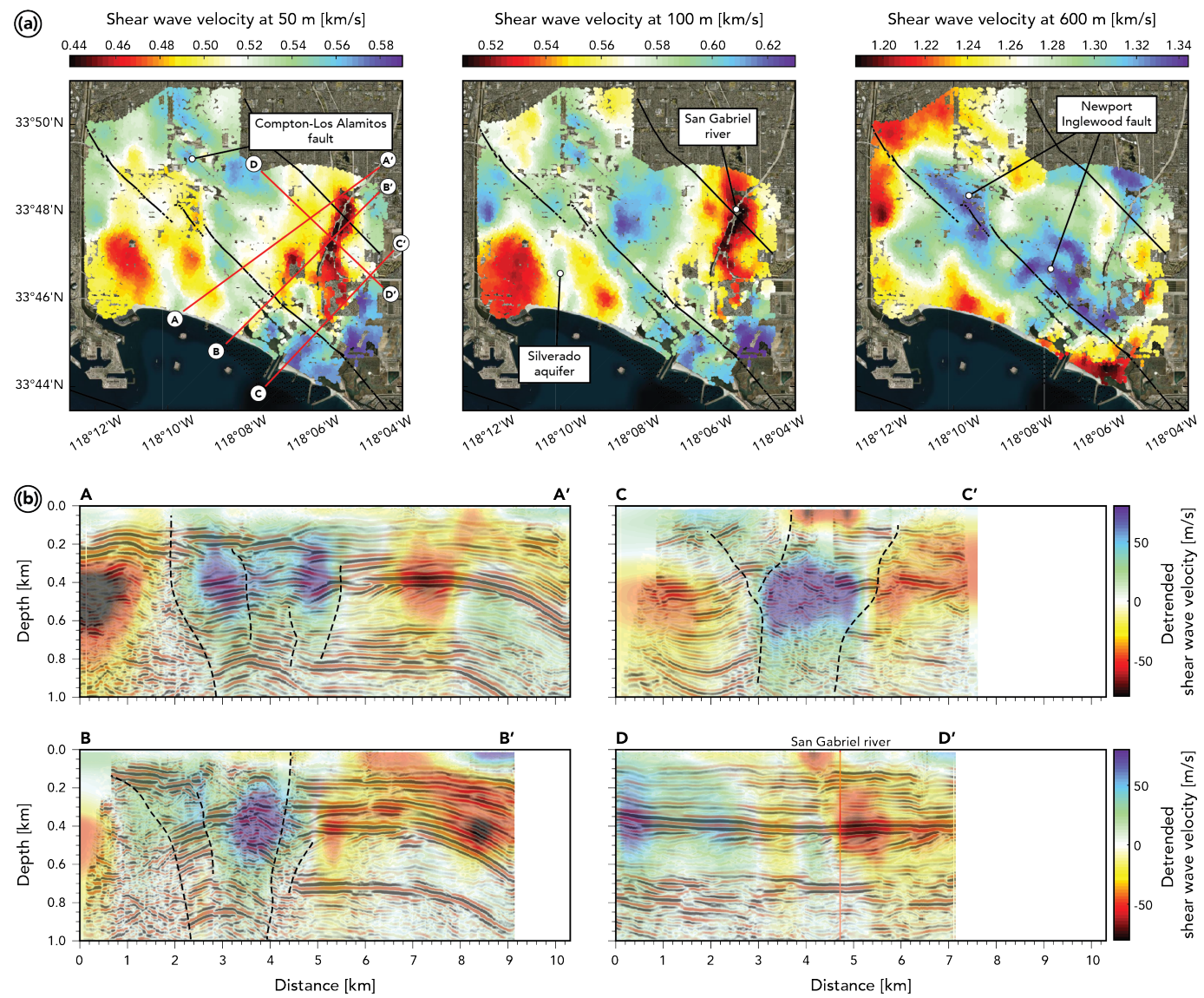

Figure 10. Depth slices and cross-sections of our velocity model. (a) Shows a depth slice our velocity model at 50-, 100-, and 600-m depths. (b) Shows 4 cross-sections of our velocity model plotted on top of the migrated reflection images that were generated by the petroleum company that carried out the Seal Beach survey. To highlight the amount of lateral variation in each of our profiles, we removed the average velocity at each depth. The black dashed lines are used to mark prominent discontinuities in the migrated images. The location of each cross-section is shown in the left-most map in (a). The migrated reflection profiles are courtesy of 3D Seismic Solutions. 

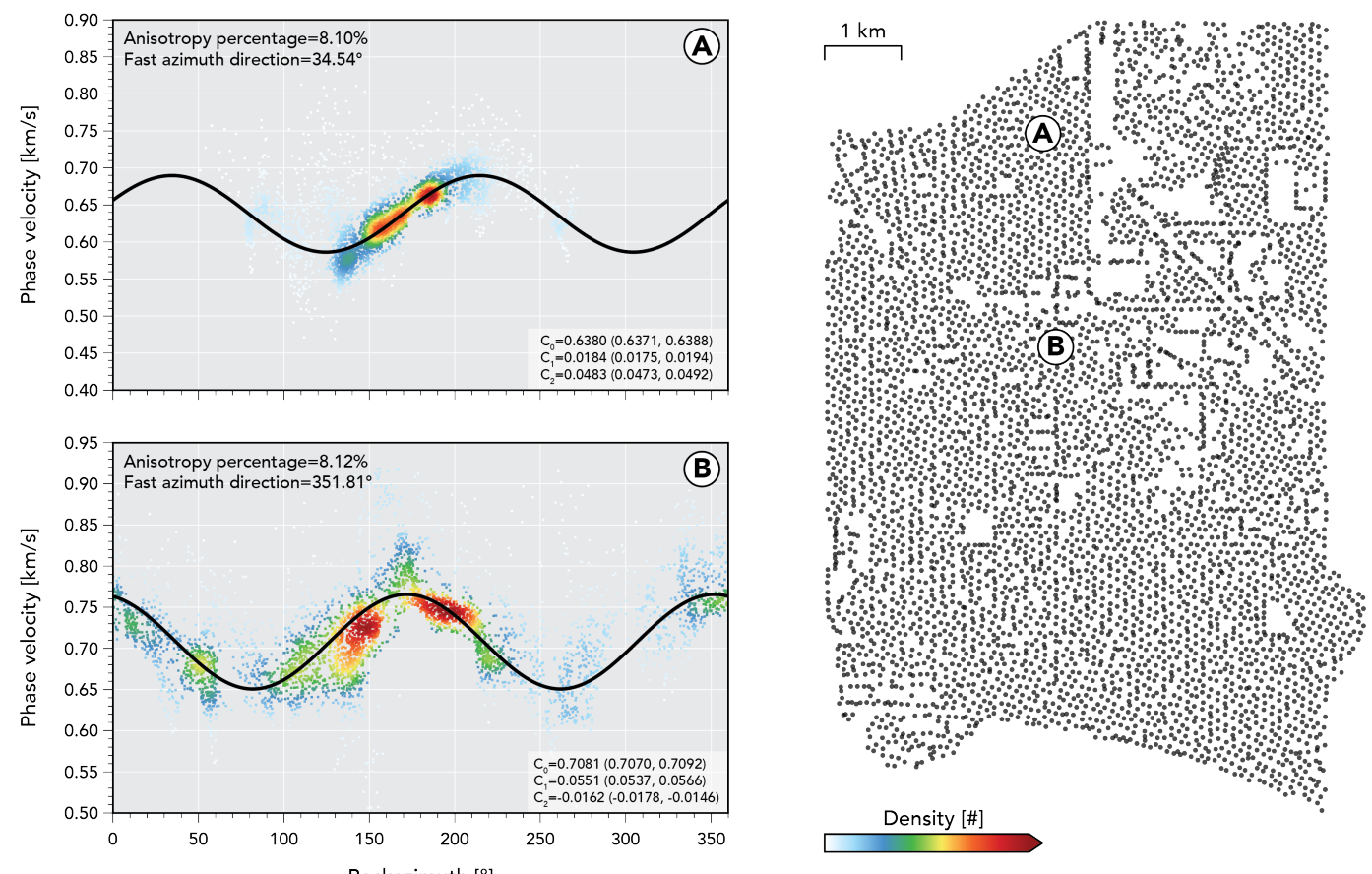

Figure 11. Example of the Rayleigh wave azimuthal dependence at 1-s period for two different locations at the Long Beach array. The color of the point cloud represents the density of the velocity measurements and the black curve marks the predicted directionality using the first three terms in Equation 6. The best fitting coefficients, along with their 95\% confidence intervals, are shown in the bottom right of each panel. 


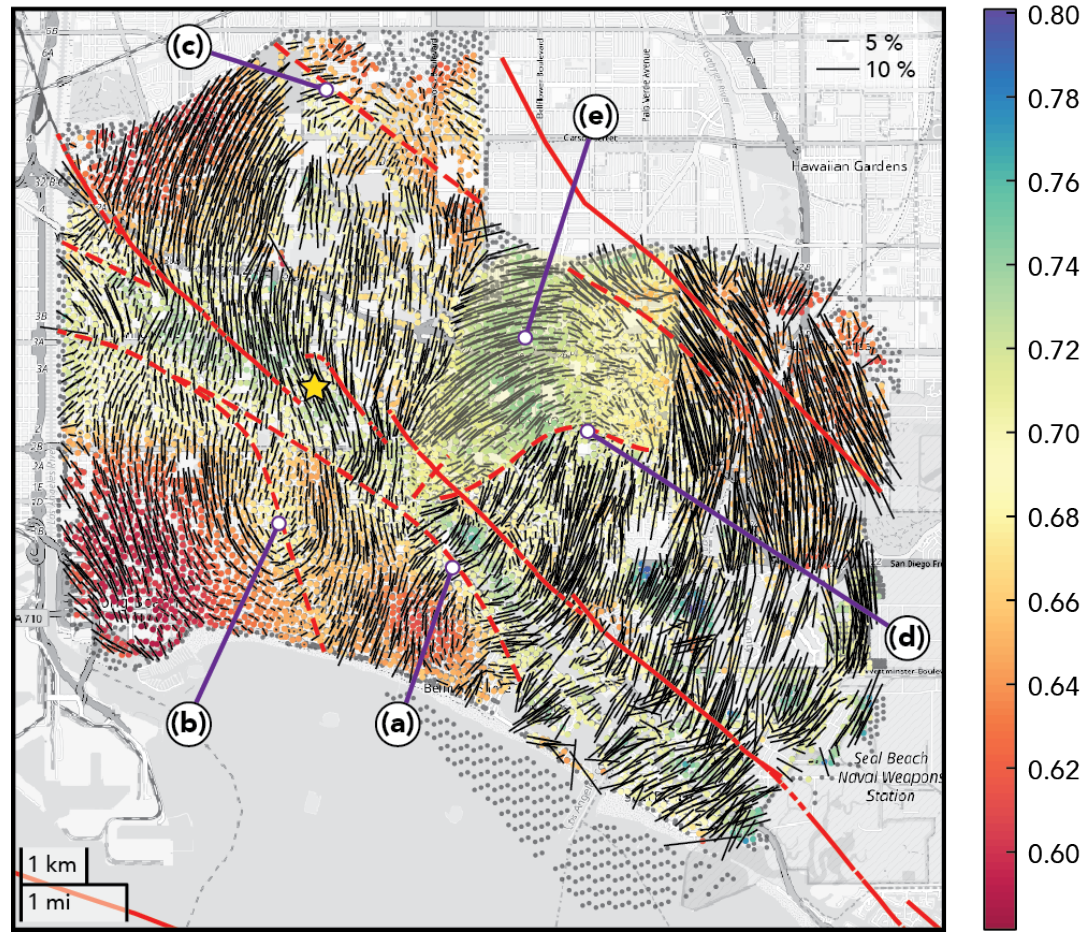

Figure 12. Anisotropy measurements for the 1-s period Rayleigh wave for the Long Beach array. The bars point to the fast direction of anisotropy and their length is proportional to the magnitude. The colored markers behind each anisotropy vector denote the isotropic velocity $\left(C_{0}\right.$ term in Equation 6). The continuous red line on the right map marks the mapped surface trace of the regional faults, whereas the dashed red lines delineate sharp changes in the anisotropy measurements that we suggest might be indicative of the presence of faults or other geologic boundaries. We associate these structures to the following: (a) NIF zone; (b) Silverado aquifer; (c) Compton-Los Alamitos Fault; (d) Garden Grove Fault; (e) Prograding clinoforms (see reflection image in Figure 8). The yellow star marks the location of Signal Hill. 

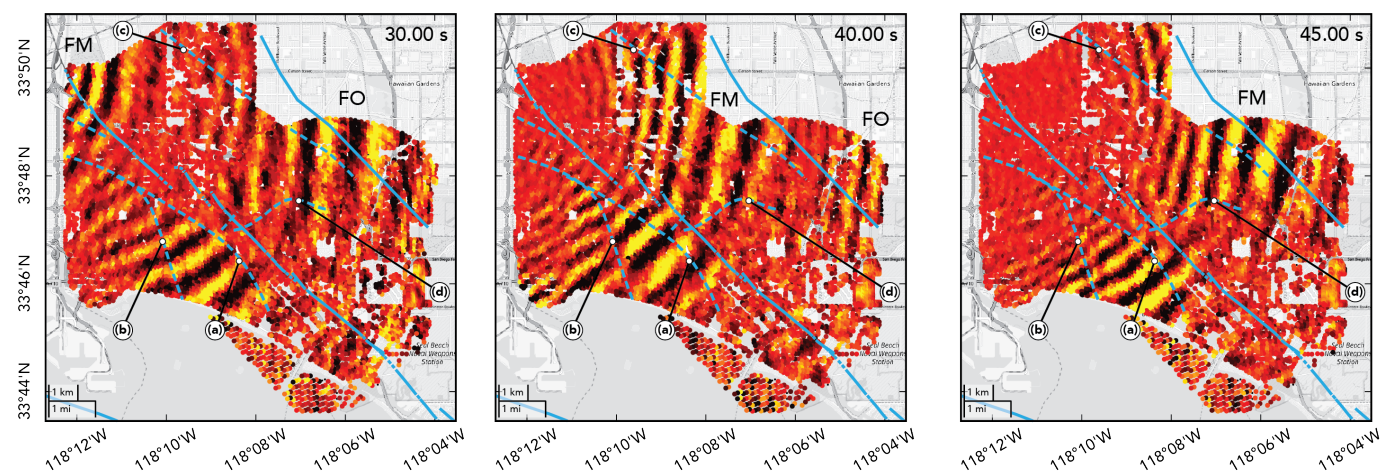

Figure 13. Wavefield snapshots of the ambient noise correlation functions using station LAF of the SCSN as a virtual source. Clear fundamental mode (FM) and first overtone (FO) surface waves can be observed. The dashed lines are the same as in Figure 12 and delineate the zones where the anisotropy measurements exhibit a drastic change in direction. The wavefield was band-passed filtered between 0.5 and $1.5 \mathrm{~Hz}$. The time-stamp of each frame is shown in the upper right side of the panels. 

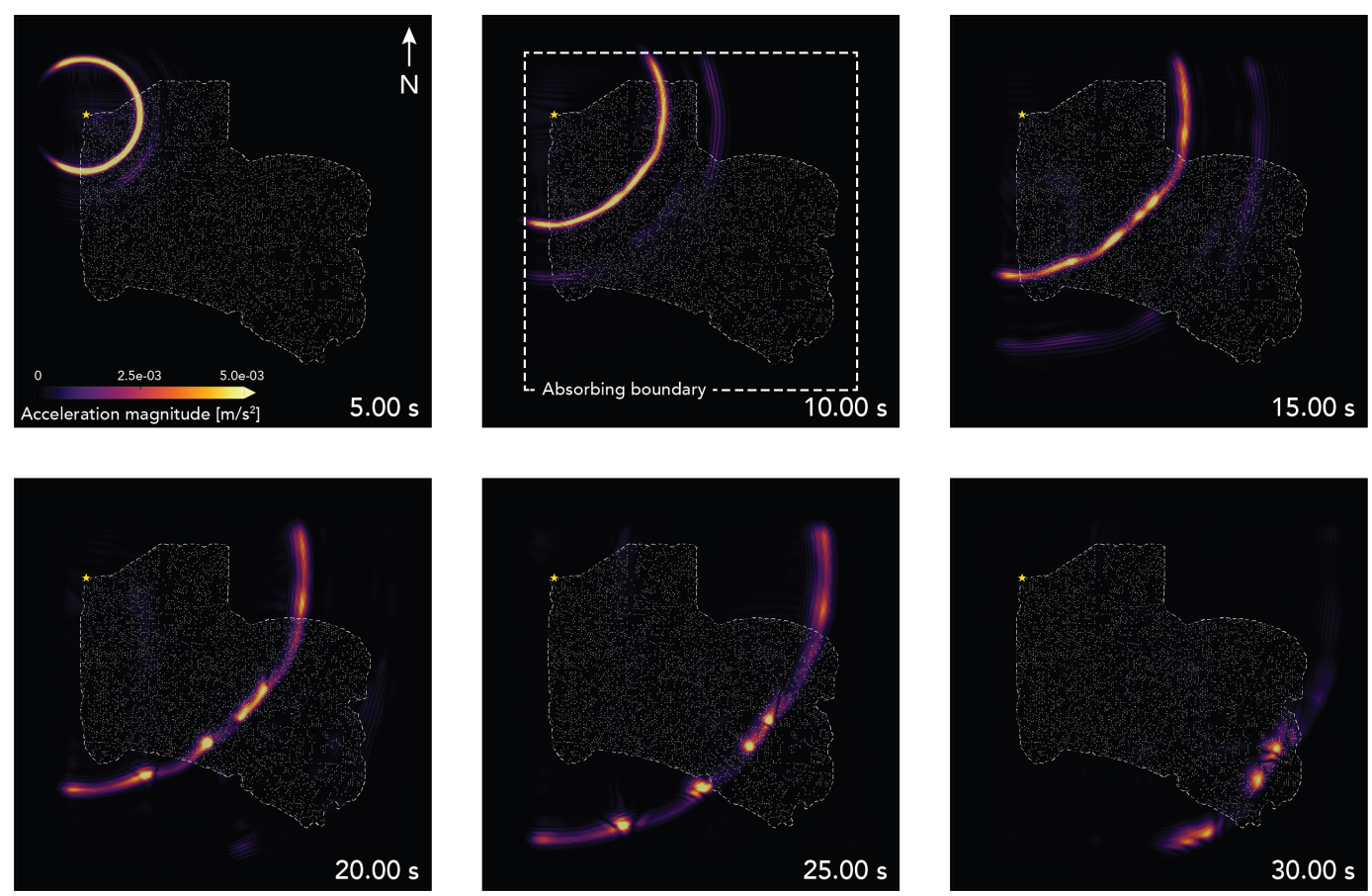

Figure 14. Snapshots of a synthetic wavefield propagated through our velocity model. The location of the source is indicated by the yellow star and corresponds to a vertical force injected by a ricker wavelet with a central frequency of $2 \mathrm{~Hz}$. Note the lateral differences in the wavefront's amplitude as it propagates away from the source. The time-stamp of each frame is shown in the lower right side of the panels. 


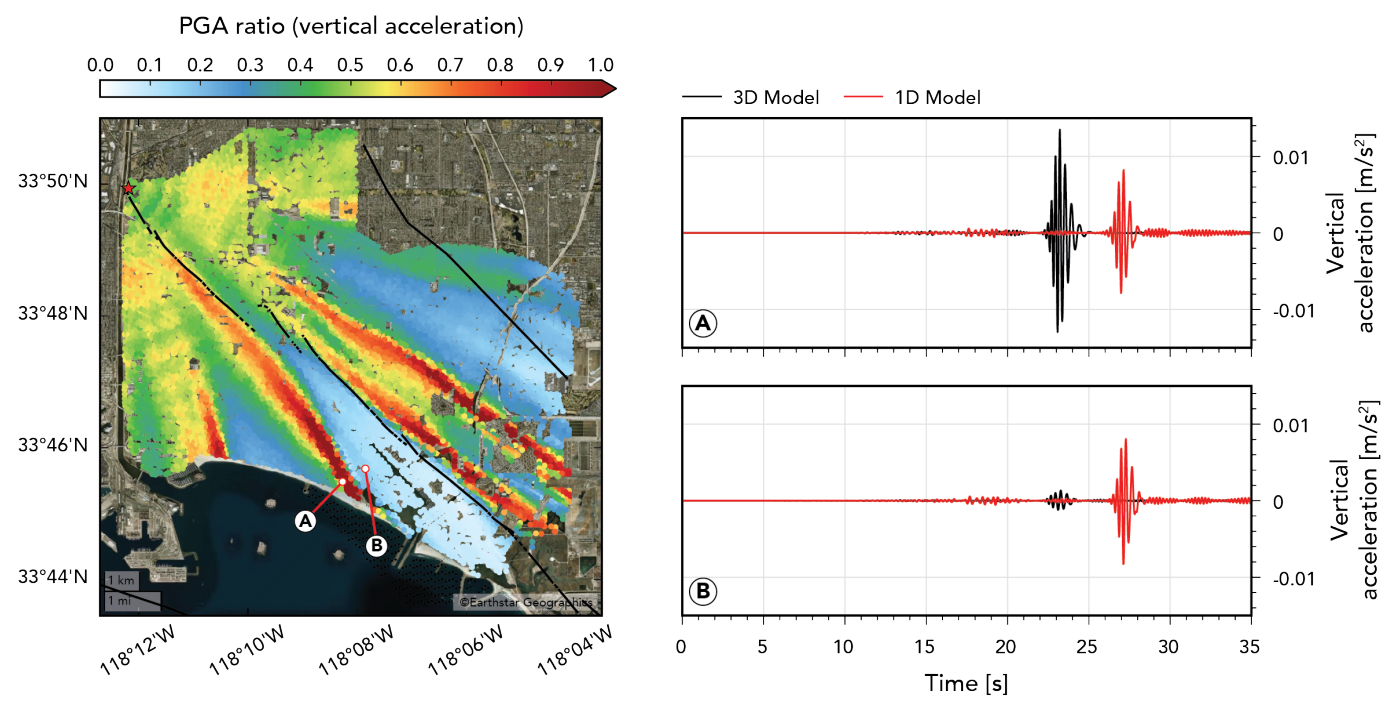

Figure 15. Map of relative amplification for the source configuration shown in Figure 14.

These values describe the expected PGA relative to the one that is obtained from a 1-D model.

The red star marks the location of the source. The right panels show the synthetic waveforms that are recorded at locations $\mathrm{A}$ and $\mathrm{B}$ of the map for both our 3-D model (black) and a 1-D model (red). Note how the structure of the NIF generates elongated zones of intense amplification that result in ground motion changes that vary for several factors on the sub-kilometer scale. The waveforms are low-passed filtered at $4 \mathrm{~Hz}$, which is the maximum frequency that is resolvable in our simulations. 


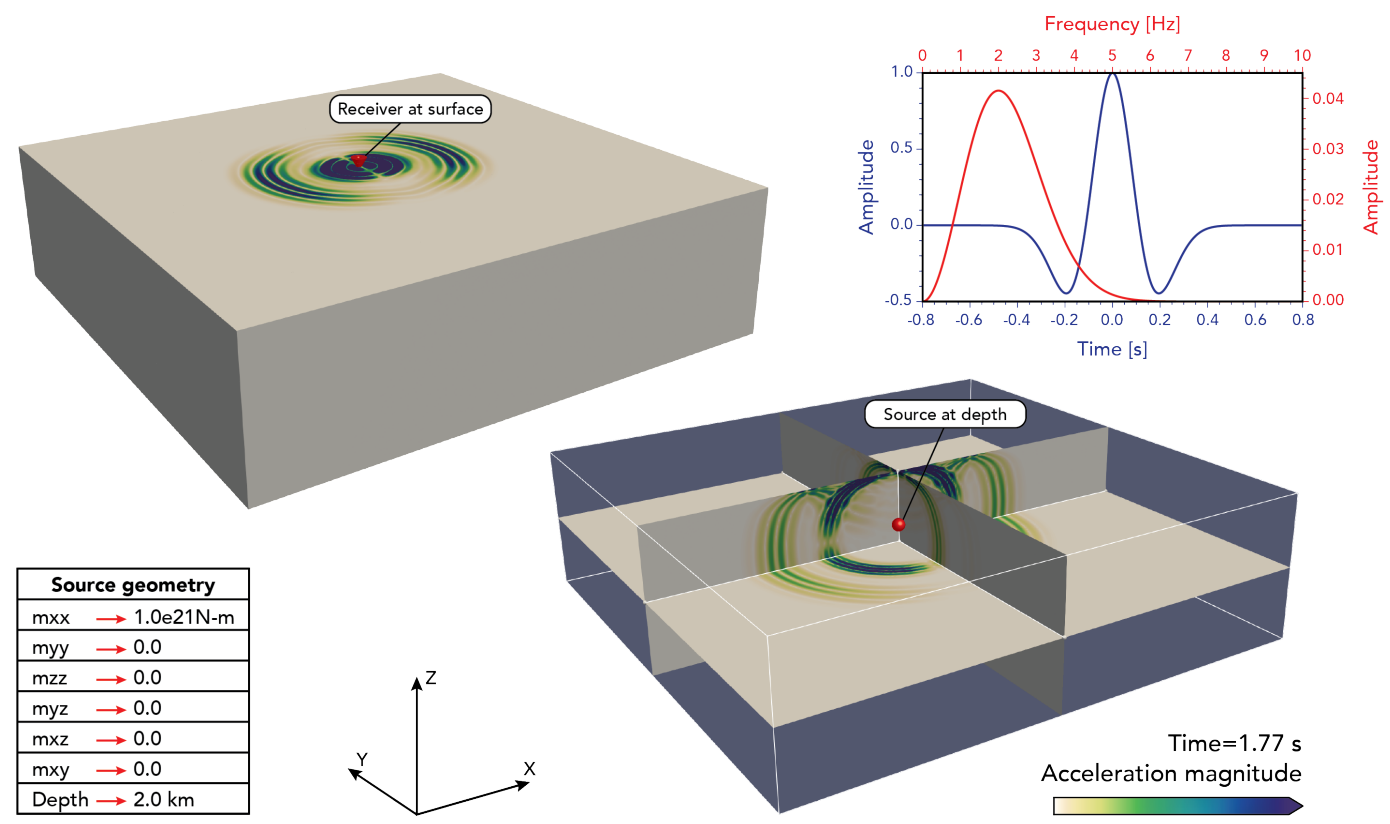

Figure 16. Experiment set-up for the estimation of the amplification factors for the case of a vertically propagating shear wave. The source wavelet, and its frequency content, are shown in the upper right panel. 


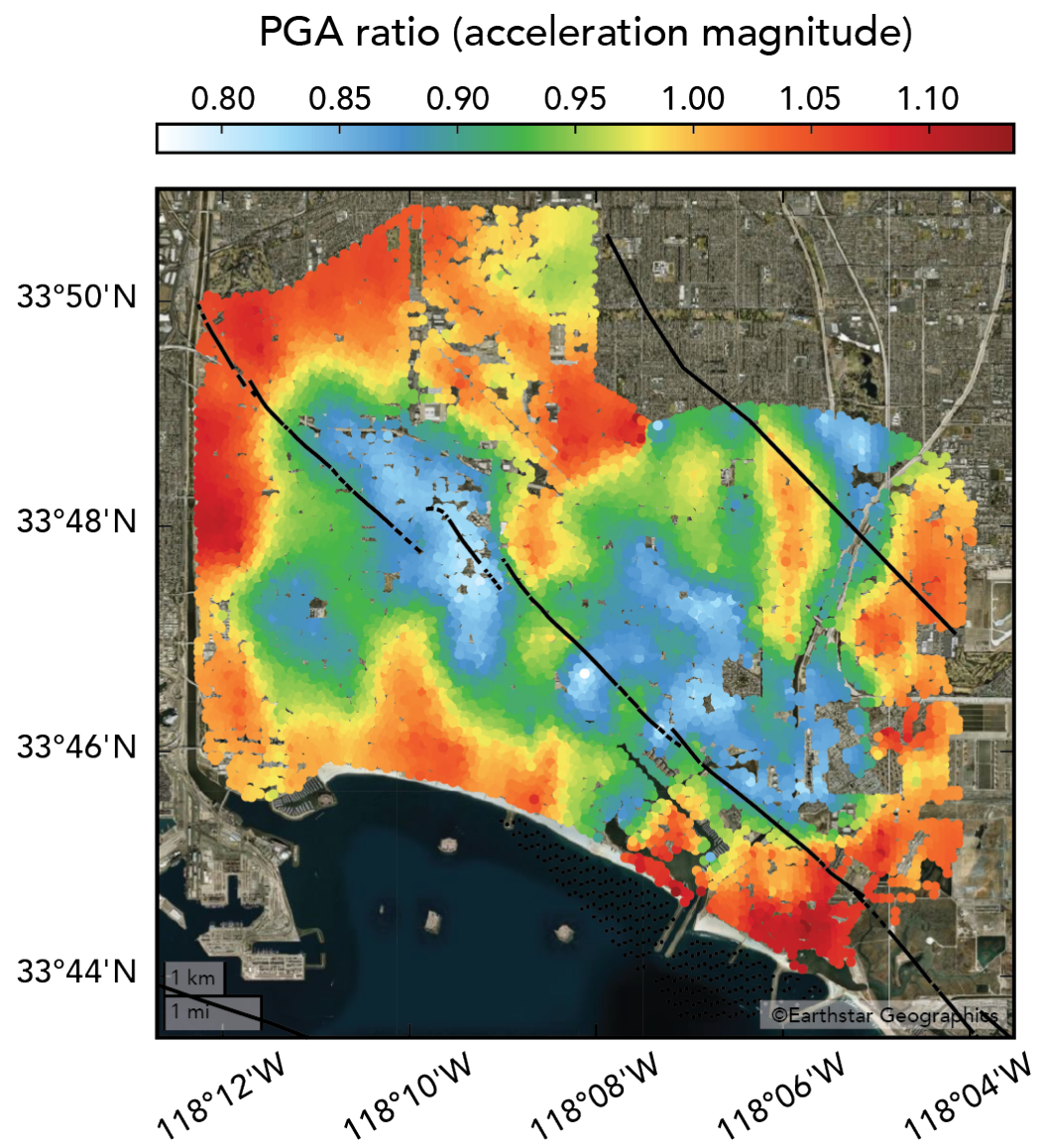

Figure 17. Map of relative amplification for the case of a vertically propagating shear wave.

These values describe the expected PGA relative to the one that is obtained from a 1-D model. 

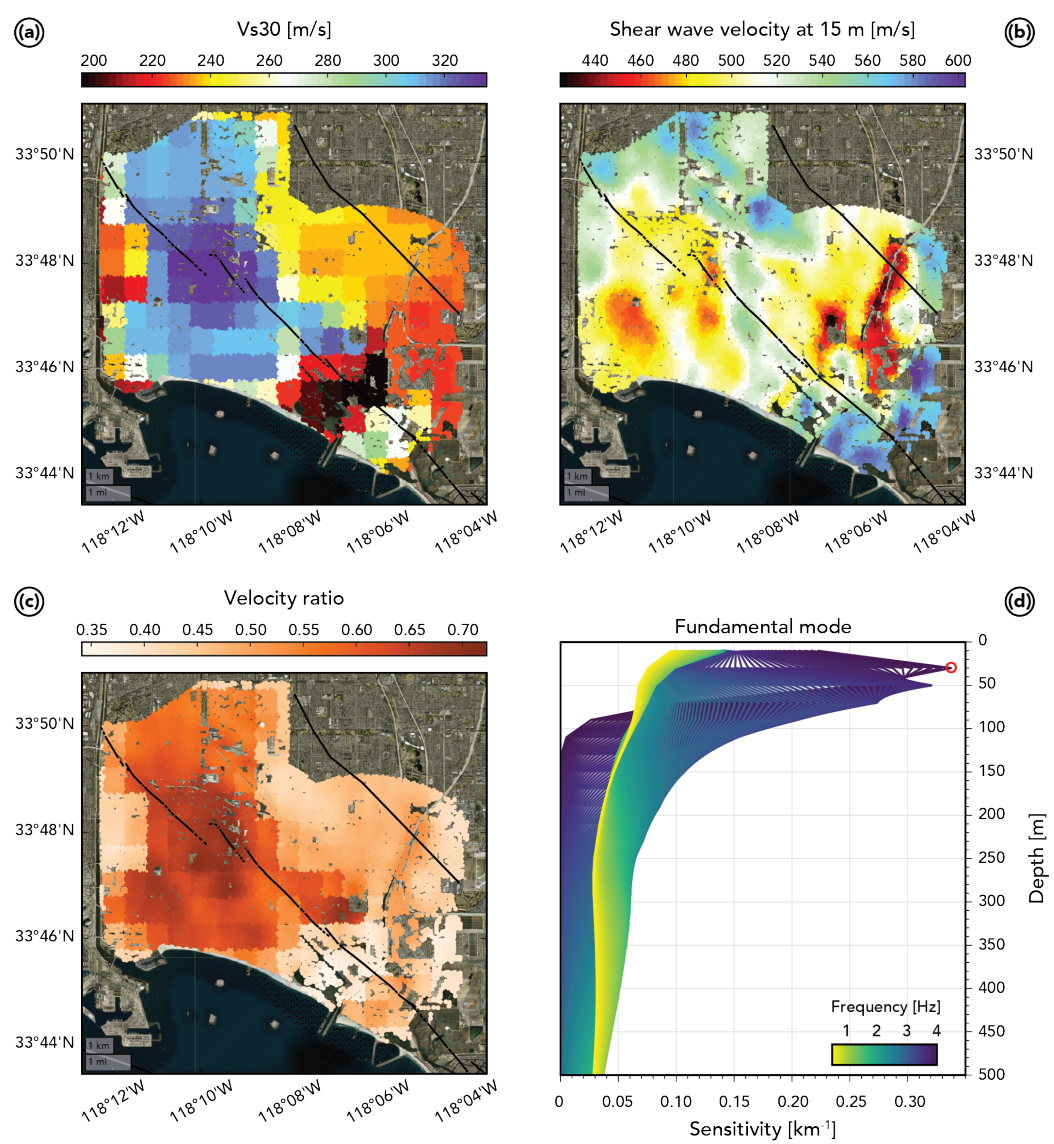

Figure 18. Comparison between the Vs30 and the velocity measurements of this investigation. (a) Shows the Vs30 values across the surveys. (b) Shows our shear wave velocity estimates at 15-m depth. (c) Shows the ratio of (a) and (b). (d) Shows the sensitivity kernels of our measurements for the frequency range of analysis. The shallowest kernel peaks at around 30-m depth (red circle).

\section{Acknowledgments}

We gratefully acknowledge NodalSeismic and Signal Hill Petroleum for allowing us to use the Long Beach and Extended Long Beach data, and LA seismic for the use of the Seal Beach data. Permission form these organizations is required to access the data. We thank Dan Hollis and Eric Campbell for facilitating the use of the seismic data. We extend our acknowledgement to Dan Gish and Steve Boljen for providing us with the migrated seismic sections of the Seal Beach survey. We also thank Michael Afanasiev and the Mondaic team for their help in setting up the numeric simulations. We are grateful to Zhe Jia for providing us with the ambient noise cross-correlations between the nodal 
instruments and the SCSN seismic station, and to Bruce Worden for providing us with the Vs30 measurements. The 3-D shear wave velocity model that is presented in this work can be downloaded from: https://doi.org/10.22002/D1.1970. This project was partially supported by NSF/EAER-1520081.

\section{References}

Afanasiev, M., Boehm, C., van Driel, M., Krischer, L., Rietmann, M., May, D. A., ... Fichtner, A. (2019). Modular and flexible spectral-element waveform modelling in two and three dimensions. Geophysical Journal International, 216(3), $1675-1692$.

Backus, G. (1970). A geometrical picture of anisotropic elastic tensors. Reviews of geophysics, 8(3), 633-671.

Barmin, M., Ritzwoller, M., \& Levshin, A. (2001). A fast and reliable method for surface wave tomography. In Monitoring the comprehensive nuclear-test-ban treaty: Surface waves (pp. 1351-1375). Springer.

Bensen, G., Ritzwoller, M., Barmin, M., Levshin, A. L., Lin, F., Moschetti, M., ... Yang, Y. (2007). Processing seismic ambient noise data to obtain reliable broad-band surface wave dispersion measurements. Geophysical Journal International, $169(3), 1239-1260$.

Bolin, R., \& Stanford, L. (1998). The northridge earthquake: community-based approaches to unmet recovery needs. Disasters, 22(1), 21-38.

Bowden, D. C., \& Tsai, V. C. (2017). Earthquake ground motion amplification for surface waves. Geophysical Research Letters, 44(1), 121-127.

Castellanos, J. C., Clayton, R. W., \& Juarez, A. (2020). Using a time-based subarray method to extract and invert noise-derived body waves at long beach, california. Journal of Geophysical Research: Solid Earth, 125(5), e2019JB018855.

Clayton, R., Heaton, T., Chandy, M., Krause, A., Kohler, M., Bunn, J., ... others (2011). Community seismic network. Annals of Geophysics, 54(6), 738-747.

Clayton, R. W. (2020). A detailed image of the continent-borderland transition beneath long beach, california. Geophysical Journal International, 222(3), 21022107.

Costa, G., Panza, G. F., Suhadolc, P., \& Vaccari, F. (1993). Zoning of the italian territory in terms of expected peak ground acceleration derived from complete 
synthetic seismograms. Journal of applied geophysics, 30(1-2), 149-160.

Crampin, S. (1994). The fracture criticality of crustal rocks. Geophysical Journal International, 118(2), 428-438.

Gardner, G., Gardner, L., \& Gregory, A. (1974). Formation velocity and densitythe diagnostic basics for stratigraphic traps. Geophysics, 39(6), 770-780.

Gish, D., \& Boljen, S. (2021). Personal Communication.

Gouédard, P., Yao, H., Ernst, F., \& van der Hilst, R. D. (2012). Surface wave eikonal tomography in heterogeneous media using exploration data. Geophysical Journal International, 191(2), 781-788.

Goulet, C. A., Haselton, C. B., Mitrani-Reiser, J., Beck, J. L., Deierlein, G. G., Porter, K. A., \& Stewart, J. P. (2007). Evaluation of the seismic performance of a code-conforming reinforced-concrete frame building - from seismic hazard to collapse safety and economic losses. Earthquake Engineering $\&$ Structural Dynamics, 36(13), 1973-1997.

Graves, R., \& Pitarka, A. (2016). Kinematic ground-motion simulations on rough faults including effects of $3 \mathrm{~d}$ stochastic velocity perturbations. Bulletin of the Seismological Society of America, 106(5), 2136-2153.

Graves, R. W. (2008). The seismic response of the san bernardino basin region during the 2001 big bear lake earthquake. Bulletin of the Seismological Society of America, 98(1), 241-252.

Haney, M. M., \& Tsai, V. C. (2017). Perturbational and nonperturbational inversion of rayleigh-wave velocities. Geophysics, 82(3), F15-F28.

Harding, T. P. (1985). Seismic characteristics and identification of negative flower structures, positive flower structures, and positive structural inversion. $A A P G$ Bulletin, 69(4), 582-600.

Harkrider, D. G. (1964). Surface waves in multilayered elastic media i. rayleigh and love waves from buried sources in a multilayered elastic half-space. Bulletin of the Seismological Society of America, 54(2), 627-679.

Inbal, A., Ampuero, J. P., \& Clayton, R. W. (2016). Localized seismic deformation in the upper mantle revealed by dense seismic arrays. Science, 354(6308), 8892.

Jia, Z., \& Clayton, R. W. (2021). Determination of near surface shear-wave velocities in the central los angeles basin with dense arrays. Journal of Geophysical 
Research: Solid Earth, e2020JB021369.

Jin, G., \& Gaherty, J. B. (2015). Surface wave phase-velocity tomography based on multichannel cross-correlation. Geophysical Journal International, 201(3), $1383-1398$.

Kohler, M., Magistrale, H., \& Clayton, R. (2003). Mantle heterogeneities and the scec reference three-dimensional seismic velocity model version 3. Bulletin of the Seismological Society of America, 93(2), 757-774.

Lee, E.-J., Chen, P., Jordan, T. H., Maechling, P. B., Denolle, M. A., \& Beroza, G. C. (2014). Full-3-d tomography for crustal structure in southern california based on the scattering-integral and the adjoint-wavefield methods. Journal of Geophysical Research: Solid Earth, 119(8), 6421-6451.

Leon, L. A., Dolan, J. F., Shaw, J. H., \& Pratt, T. L. $\quad$ (2009). Evidence for large holocene earthquakes on the compton thrust fault, los angeles, california. Journal of Geophysical Research: Solid Earth, 114(B12).

Li, Z., Peng, Z., Hollis, D., Zhu, L., \& McClellan, J. (2018). High-resolution seismic event detection using local similarity for large-n arrays. Scientific reports, 8(1), $1-10$.

Licciardi, A., Eken, T., Taymaz, T., Agostinetti, N. P., \& Yolsal-Çevikbilen, S. (2018). Seismic anisotropy in central north anatolian fault zone and its implications on crustal deformation. Physics of the Earth and Planetary Interiors, 277, 99-112.

Lin, F.-C., Li, D., Clayton, R. W., \& Hollis, D. (2013). High-resolution 3d shallow crustal structure in long beach, california: Application of ambient noise tomography on a dense seismic array. Geophysics, 78(4), Q45-Q56.

Lin, F.-C., Moschetti, M. P., \& Ritzwoller, M. H. (2008). Surface wave tomography of the western united states from ambient seismic noise: Rayleigh and love wave phase velocity maps. Geophysical Journal International, 173(1), $281-298$.

Liu, G., Persaud, P., \& Clayton, R. W. (2018). Structure of the northern los angeles basins revealed in teleseismic receiver functions from short-term nodal seismic arrays. Seismological Research Letters, 89(5), 1680-1689.

Magistrale, H., Day, S., Clayton, R. W., \& Graves, R. (2000). The scec southern california reference three-dimensional seismic velocity model version 2. Bulletin of 
the Seismological Society of America, 90(6B), S65-S76.

McMechan, G. A., \& Yedlin, M. J. (1981). Analysis of dispersive waves by wave field transformation. Geophysics, 46(6), 869-874.

Moschetti, M., Ritzwoller, M., \& Shapiro, N. (2007). Surface wave tomography of the western united states from ambient seismic noise: Rayleigh wave group velocity maps. Geochemistry, Geophysics, Geosystems, 8(8).

Muir, J. B., \& Tsai, V. C. (2020). Geometric and level set tomography using ensemble kalman inversion. Geophysical Journal International, 220(2), 967-980.

Nakata, N., Chang, J. P., Lawrence, J. F., \& Boué, P. (2015). Body wave extraction and tomography at long beach, california, with ambient-noise interferometry. Journal of Geophysical Research: Solid Earth, 120(2), 1159-1173.

Nicolson, H., Curtis, A., Baptie, B., \& Galetti, E. (2012). Seismic interferometry and ambient noise tomography in the british isles. Proceedings of the Geologists' Association, 123(1), 74-86.

Olsen, K., Day, S., Dalguer, L., Mayhew, J., Cui, Y., Zhu, J., ... others (2009). Shakeout-d: Ground motion estimates using an ensemble of large earthquakes on the southern san andreas fault with spontaneous rupture propagation. Geophysical Research Letters, 36(4).

Ozacar, A. A., \& Zandt, G. (2009). Crustal structure and seismic anisotropy near the san andreas fault at parkfield, california. Geophysical Journal International, 178(2), 1098-1104.

Poland, J. F. (1956). Ground-water geology of the coastal zone, long beach-santa ana area, california (Vol. 1109). US Government Printing Office.

Ritzwoller, M. H., Lin, F.-C., \& Shen, W. (2011). Ambient noise tomography with a large seismic array. Comptes Rendus Geoscience, 343(8-9), 558-570.

Rodgers, A. J., Pitarka, A., Petersson, N. A., Sjögreen, B., \& McCallen, D. B.

(2018). Broadband (0-4 hz) ground motions for a magnitude 7.0 hayward fault earthquake with three-dimensional structure and topography. Geophysical Research Letters, 45(2), 739-747.

Shapiro, N. M., \& Campillo, M. (2004). Emergence of broadband rayleigh waves from correlations of the ambient seismic noise. Geophysical Research Letters, $31(7)$.

Shapiro, N. M., Campillo, M., Stehly, L., \& Ritzwoller, M. H. (2005). High- 
resolution surface-wave tomography from ambient seismic noise.

Science, 307(5715), 1615-1618.

Shaw, J. H., Plesch, A., Tape, C., Suess, M. P., Jordan, T. H., Ely, G., .. others (2015). Unified structural representation of the southern california crust and upper mantle. Earth and Planetary Science Letters, 415, 1-15.

Smith, M. L., \& Dahlen, F. (1973). The azimuthal dependence of love and rayleigh wave propagation in a slightly anisotropic medium. Journal of Geophysical Research, 78(17), 3321-3333.

Stover, C. W., \& Coffman, J. L. (1993). Seismicity of the united states, 1568-1989 (revised). US Government Printing Office.

Taborda, R., Azizzadeh-Roodpish, S., Khoshnevis, N., \& Cheng, K. (2016). Evaluation of the southern california seismic velocity models through simulation of recorded events. Geophysical Journal International, 205(3), 1342-1364.

Tape, C., Liu, Q., Maggi, A., \& Tromp, J. (2009). Adjoint tomography of the southern california crust. Science, 325(5943), 988-992.

Thompson, E., Wald, D. J., \& Worden, C. (2014). Avs 30 map for california with geologic and topographic constraints. Bulletin of the Seismological Society of America, 104(5), 2313-2321.

VanDecar, J., \& Crosson, R. (1990). Determination of teleseismic relative phase arrival times using multi-channel cross-correlation and least squares. Bulletin of the Seismological Society of America, 80(1), 150-169.

Wald, D. J., \& Graves, R. W. (1998). The seismic response of the los angeles basin, california. Bulletin of the Seismological Society of America, 88(2), 337-356.

Wang, X., \& Zhan, Z. (2020). Moving from 1-d to 3-d velocity model: automated waveform-based earthquake moment tensor inversion in the los angeles region. Geophysical Journal International, 220(1), 218-234.

Wesnousky, S. G. (2005). The san andreas and walker lane fault systems, western north america: Transpression, transtension, cumulative slip and the structural evolution of a major transform plate boundary. Journal of Structural Geology, 27(8), 1505-1512.

Wills, C., Petersen, M., Bryant, W., Reichle, M., Saucedo, G., Tan, S., ... Treiman, J. (2000). A site-conditions map for california based on geology and shear-wave velocity. Bulletin of the Seismological Society of America, 90(6B), S187-S208. 
Wood, H. O. (1933). Preliminary report on the long beach earthquake. Bulletin of the Seismological Society of America, 23(2), 43-56.

Wright, T. L. (1991). Structural geology and tectonic evolution of the los angeles basin, california: Chapter 3: Part 1.

Yang, L., Liu, X., \& Beroza, G. C. (2021). Revisiting evidence for widespread seismicity in the upper mantle under los angeles. Science Advances, 7(4), eabf2862.

Yang, W., \& Hauksson, E. (2013). The tectonic crustal stress field and style of faulting along the pacific north america plate boundary in southern california. Geophysical Journal International, 194(1), 100-117.

Yao, H., van Der Hilst, R. D., \& De Hoop, M. V. (2006). Surface-wave array tomography in se tibet from ambient seismic noise and two-station analysis-i. phase velocity maps. Geophysical Journal International, 166(2), 732-744.

Zhong, M., \& Zhan, Z. (2020). An array-based receiver function deconvolution method: methodology and application. Geophysical Journal International, 222(1), 1-14. 